\title{
Thermoelectric properties of the (an-)isotropic QGP in magnetic fields
}

\author{
He-Xia Zhang ${ }^{1, \mathrm{a}}$, Jin-Wen Kang ${ }^{1}$, Ben-Wei Zhang ${ }^{1,2, \mathrm{~b}}{ }_{\mathbb{C}}$ \\ ${ }^{1}$ Key Laboratory of Quark and Lepton Physics (MOE) and Institute of Particle Physics, Central China Normal University, Wuhan 430079, China \\ ${ }^{2}$ Institute of Quantum Matter, South China Normal University, Guangzhou 510006, China
}

Received: 24 January 2021 / Accepted: 5 July 2021 / Published online: 17 July 2021

(C) The Author(s) 2021

\begin{abstract}
The Seebeck effect and the Nernst effect, which reflect the appearance of electric fields along $x$-axis and along $y$-axis $\left(E_{x}\right.$ and $E_{y}$ ), respectively, induced by the thermal gradient along $x$-axis, are studied in the QGP at an external magnetic field along $z$-axis. We calculate the associated Seebeck coefficient $\left(S_{x x}\right)$ and Nernst signal $(N)$ using the relativistic Boltzmann equation under the relaxation time approximation. In an isotropic QGP, the influences of magnetic field $(B)$ and quark chemical potential $\left(\mu_{q}\right)$ on these thermoelectric transport coefficients are investigated. In the presence (absence) of weak magnetic field, we find $S_{x x}$ for a fixed $\mu_{q}$ is negative (positive) in sign, indicating that the dominant carriers for converting heat gradient to electric field are negatively (positively) charged quarks. The absolute value of $S_{x x}$ decreases with increasing temperature. Unlike $S_{x x}$, the sign of $N$ is independent of charge carrier type, and its thermal behavior displays a peak structure. In the presence of strong magnetic field, due to the Landau quantization of transverse motion of (anti-)quarks perpendicular to magnetic field, only the longitudinal Seebeck coefficient $\left(S_{z z}\right)$ exists. Our results show that the value of $S_{z z}$ at a fixed $\mu_{q}$ in the lowest Landau level (LLL) approximation always remains positive. Within the effect of high Landau levels, $S_{z z}$ exhibits a thermal structure similar to that in the LLL approximation. As the Landau level increases further, $S_{z z}$ decreases and even its sign changes from positive to negative. The computations of these thermoelectric transport coefficients are also extended to a medium with momentum-anisotropy induced by initial spatial expansion as well as strong magnetic field.
\end{abstract}

\footnotetext{
a e-mail: zhanghexia@mails.ccnu.edu.cn

b e-mail: bwzhang@mail.ccnu.edu.cn (corresponding author)
}

\section{Introduction}

Quantum chromodynamics (QCD) is the fundamental theory of the strong interaction, and the Lattice QCD calculations have predicted a crossover phase transition from the hadronic matter to the quark-gluon plasma (QGP) can be realized with the increase of temperature at the small or vanishing baryon chemical potential [1,2]. Heavy-ion collisions (HICs) experiments with very large center-of-mass colliding energies, e.g., the relativistic heavy-ion collision (RHIC) at BNL and the large hadron collision (LHC) at CERN also provide an opportunity to deeply exploring the phase structure and the transport properties of strongly interacting matter at the extreme conditions. In the non-central HICs, the presence of an enormous magnetic field in the direction perpendicular to the reaction plane is expected [3]. The theoretical estimate of field strength at primary stage of collisions can reach $e B \sim m_{\pi}^{2} \sim 0.02 \mathrm{GeV}^{2}$ for RHIC and $e B \sim 15 m_{\pi}^{2} \sim 0.3$ $\mathrm{GeV}^{2}$ for LHC [4-9]. And this magnetic field can persist long-lived due to the presence of electrical conductivity of medium [9-11]. In the past years, a variety of novel insights of strongly interacting matter induced by strong magnetic background field have sparkled considerable research, such as chiral magnetic effect $[8,12,13]$, the chiral magnetic wave $[14,15]$, inverse magnetic catalysis [16-22], and the heavy quark transport [23-29], etc. Thus, investigating the magnetic field-induced phenomenological consequences and the effect of magnetic field on transport properties can provide a comprehensive understanding of the complex QCD matter.

Transport coefficients, characterizing the dynamical evolution of system, play a crucial role to probe the strongly interacting matter. Recently, numerous works have been devoted to studying the effects of magnetic field on transport coefficients in QCD matter. Due to the uncertainty of the realistic magnitude of magnetic field produced in the initial stage of HICs, we only consider that the magnetic field is constant and homogeneous in present work. In the pres- 
ence of a magnetic field oriented along $z$-axis the Landau gauge $A_{\mu}=(0,0,-B x, 0)$ ( $B$ is the strength of an artificial magnetic field) is chosen as usual, where $A_{\mu}$ denotes the electromagnetic potential. By solving the Dirac equation of motion, the dispersion relation for light (anti-)quarks in the QGP is obtained quantum-mechanically as [30-32] $\epsilon_{f, l}=\sqrt{p_{z}^{2}+m_{f}^{2}+2 l\left|q_{f} e B\right|}(l=0,1, \ldots$ are the quantum numbers of the Landau energy levels; $m_{f}$ is the current mass for $f$ th flavor (anti-)quarks; $p_{z}$ is the momentum of charged particle along the direction of magnetic field; $q_{f} e$ is the electric charge of $f$ th flavor quarks.). In the literature, the ranges of magnetic field can be roughly categorized into three scenarios: the weak magnetic case or classical case, the strong magnetic field case or higher Landau levels (hLLs) case, the strong magnetic field limit case or lowest Landau level (LLL) case, which are implemented by different scale hierarchies. In the weak field case within the regime $g^{2} T^{2} \ll e B$ ( $g$ is the QCD charge, $\left.g \ll 1\right)$, the temperature acts as the dominant scale in the presence of magnetic field, the quantum effect due to Landau quantization is not included, the magnetic field effect can be seen at the classical level in the so-called cyclotron motion of charged particle. Furthermore, we reasonably assume that the scattering mechanism of partons and thermodynamics in the weakly magnetized medium are unaffected by the presence of magnetic field. With the increase of $B$, in the strong magnetic field case within the regime $e B \gg g^{2} T^{2}$ introduced in Ref. [33], the quantum effect is increasingly obvious, the Landau quantization of the cyclotron motion is seen at the quantum level and the kinetic energy of quarks gets discreted into Landau levels. As the magnetic field increases further, in the extreme magnetic field limit with the scale hierarchy $e B \gg T^{2}$, the magnitude of magnetic field is sufficiently greater than other energy scales in the thermal medium, the transition from the LLL to the hLLs requires a large energy gap $\sqrt{e B}$ to excite. Consequently, the contribution from the hLLs are neglected due to the suppression of the Boltzmann factor $\sim e^{-\sqrt{2 l e B} / T}$, only keeps the contributions from the LLL. In the context of weak field, electrical conductivity of QGP recently have been computed using the kinetic theory [34] and quasi-particle models [35,36]. And the systematic studies of shear viscosity at weak field have been done within perturbative QCD in leading log [37]. These observables have also been investigated in hadronic matter at weak magnetic field within hadron resonance gas (HRG) model [38,39]. At the strong magnetic field within the LLL approximation, electrical conductivity along the direction of magnetic field in the QGP has been estimated using diagrammatic method [40], perturbative QCD approach [41], and effective quasi-particle model [42]. In the LLL approximation, the effect of magnetic field on other observables, such as viscosities [43-47], heavy quark complex potential [48] diffusion coefficients of heavy quark [23,27], heavy quark collisional energy loss [49], the properties of quarkonium states [50] and jet quenching parameter [51] also have been studied. Furthermore, the effect of hLLs on various transport coefficients has been investigated recently in Refs. [27,33,47,52].

Besides aforementioned common transport coefficients, some attention recently has been turned to the studies of electromagnetic and thermoelectric effects such as the Hall effect, the Seebeck effect and the Nernst effect, which are also fundamental to understand the electrical transport properties of QCD matter. The Hall effect describes the generation of a transverse electric field in an electric current-carrying conductor when a uniform magnetic field perpendicular to the direction of current is applied, which is usually studied in solid materials. In the hot QCD matter, due to the significant initial velocity of charged particles along the beam direction is perpendicular to the produced magnetic field created in non-central HICs, the Lorentz force can result in an electric current normal to both the initial velocity of charged particles and the magnetic field, which indicates the investigation of the Hall effect in QCD matter is reasonable. Actually, the conductivity associated this Hall current, the Hall conductivity, in the baryon rich hadronic matter as well as the QGP has been estimated already using the kinetic theory $[34,36,38]$. The Hall component of shear viscosity has also recently been studied in holographic model [53]. Furthermore, in the presence of magnetic field, a current of charge carriers can be deflected, whether it is an electrical current or a thermal current. When a temperature gradient along $x$ axis $\left(\nabla_{x} T\right)$ exists in a conducting medium, a corresponding electric field $\left(E_{x}\right)$ can arise. By applying an external magnetic field along $z$-axis, the thermal current of charge carriers generated by the temperature gradient along $x$-axis can be deflected to $y$-axis, resulting in an electric field along $y$-axis $\left(E_{y}\right)$. The appearance of $E_{x}$ and $E_{y}$ due to the thermal gradient along $x$-axis is called the Seebeck effect and the Nernst effect, respectively. Accordingly, the proportionality constants, $\frac{E_{x}}{\nabla_{x} T}$ and $\frac{E_{y}}{\nabla_{x} T}$, in zero-current condition are called the Seebeck coefficient $\left(S_{x x}\right)$ and the Nernst signal ( $N$ or $S_{x y}$ ). The strength of $S_{x x}$ and $N$ reflects the efficiency of the thermoelectric materials regarding the conversion of heat into electrical power. In condensed matter physics, the Seebeck effect and the Nernst effect have been studied in various solid state matters, such as semiconductors [54], Bismuth [55], graphene [56-58] and Weyl semimetal [59,60]. The Seebeck coefficient and the Nernst coefficient have also been estimated in hot and dense hadronic matter at zero magnetic field [61] as well as at nonzero magnetic field [62]. The Nernst effect in a strongly correlated system at finite magnetic field has also been studied by the gauge gravity duality [63]. Very recently, the Seebeck coefficient of QGP in the LLL approximation has been computed using quasi- 
particle model [64]. To the best of our knowledge, there is no estimation of the Seebeck effect and the Nernst effect for QGP in the weak magnetic field case. Hence, this provides one of main motivations for the phenomenological research in this paper. Conventionally, an ideal assumption that the constituents of QGP or of hadronic matter are isotropic in momentum space has been employed in most existing estimations of thermoelectric coefficients. However, due to the geometry of primary fireball generated in HICs is asymmetric, the different pressure gradients along different directions make the expansion along the beam direction (denoted by \|) more rapid than that along the directions perpendicular to beam direction (denoted by $\perp$ ), i.e. $p_{\|} \ll p_{\perp}[65-67,72]$. The presence of shear viscosity also can contribute to such momentum-space anisotropy, thus the anisotropy can survive a long time [72]. There are some studies to explore the influence of momentum-anisotropy induced by the initial spatial expansion on various transport coefficients [6567], collective excitations of hot QCD medium [68-71] and quarkonium bound state [72-76]. Apart from the initial spatial expansion-driven momentum anisotropy, the momentum anisotropy also can be induced by strong magnetic field. As mentioned earlier, in the strong magnetic field the motion of charged particles perpendicular to the direction of magnetic field (denoted by $\perp$ ) can be quantum-mechanically restricted due to Landau quantization, the dynamic motion is mainly along the direction of magnetic field (denoted by $\|$ ), i.e., $p_{\perp} \ll p_{\|}$. Recently, the influence of this anisotropy induced by strong field on common transport coefficients has also been analyzed in Refs. [77,78]. Hence, it is also of interest to study how the momentum anisotropy induced by initial spatial expansion and strong magnetic field affects the quantitative and qualitative features of thermoelectric coefficients in the QGP.

In present work, we first calculate the Seebeck coefficient $\left(S_{x x}\right)$ and the Nernst signal $(N)$ in an (an-)isotropic QGP at the weak magnetic field case within the hierarchy of scale $e B \ll g^{2} T^{2}$. Next, we calculate the longitudinal Seebeck coefficient $\left(S_{z z}\right)$ in the (an-)isotropic QGP under the LLL approximation within the hierarchy of scales $\alpha_{s} e B \ll T^{2} \ll$ $e B$ introduced by Fukushima et al. [23]. In the strong magnetic field, the Debye mass from quark-loops is proportional to $\sqrt{\alpha_{s, B} e B}$ ( $\alpha_{s, B}$ is magnetic field-dependent QCD running coupling constant) $[16,79,80]$. The first inequality indicates that the self-energy corrections to hard LLL (anti-)quarks and gluons can be reasonably neglected in leading order of perturbative QCD calculation. Different to the traditional binary scatterings, due to the dimensional reduction of (anti-)quarks in the presence of strong magnetic field, the dimensional mismatch between (anti-)quarks and gluons leads to the novel scattering process, i.e., the quark-antiquark pair to gluon is possible $[10,41]$. In the LLL approximation, we consider two kind of scattering processes, namely, quark-antiquark pair to gluon $2 \rightarrow 1$ process and usual quark-antiquark $t$ channel $2 \rightarrow 2$ process, where the small current (anti-)quark mass cannot be ignored because the scatterings are forbidden in massless limit according to the chirality conservation [81]. When the magnetic field is not so large within the regime $g^{2} T^{2} \ll e B$, the contribution from hLLs needs to be considered in the computation of $S_{z z}$. Note that in this work the magnetic field is regarded as an external degree of freedom, namely, we neglect the back reaction of medium on magnetic field.

The paper is organized as follows. In Sect. 2, we derive the general formulas of thermoelectric and electric conductivity tensors in an (an-)isotropic medium at the weak magnetic field by solving the relativistic Boltzmann equation under the relaxation time approximation (RTA). And the general expressions of the Seebeck coefficient and the Nernst signal are presented. In Sect. 3, using the same methodological in Sect. 2, we also deduce the formulas of both longitudinal tensors and the longitudinal Seebeck coefficient, in the strong magnetic field with the Landau quantization. In Sect. 4, the generalized expression of the thermal relaxation times related to quark chemical potential and anisotropic parameter at different magnetic field regimes are given. In Sect. 5, we discuss the qualitative and quantitative features of thermoelectric transport coefficients. In Sect. 6, we present a summary and provide an outlook for the future. More detailed derivation of the relaxation time in zero magnetic field and in the LLL approximation can be found in Appendix A and Appendix $\mathrm{B}$, respectively.

\section{Thermoelectric coefficients in an (an-)isotropic medium at weak magnetic field}

It is sufficient to calculate the thermoelectric coefficients using the kinetic theory approach. At weak magnetic field within the hierarchy of scale $e B \ll g^{2} T^{2}$, the phase space and the single particle energy are intact by magnetic field through the Landau quantization. The magnetic field enters through the cyclotron frequency of the charged particles as in classical picture. Accordingly, the dynamic evolution of a single particle distribution function $f_{a}$ in the uniform electric field $\mathbf{E}$ and magnetic field $\mathbf{B}$ can be determined by the relativistic Boltzmann equation [34]

$$
\frac{\partial f_{a}}{\partial t}+\mathbf{v}_{a} \cdot \nabla f_{a}+e_{a}\left[\mathbf{E}+\mathbf{v}_{a} \times \mathbf{B}\right] \cdot \frac{\partial f_{a}}{\partial \mathbf{p}}=C\left[f_{a}\right] .
$$

where, $e_{a}=q_{a} e, q_{a}$ and $\mathbf{v}_{a} \equiv \frac{d \epsilon_{a}}{d}=\mathbf{p} / \epsilon_{a}$ are fractional charged value and the velocity for particle species $a$, respectively. $\epsilon_{a}=\sqrt{\mathbf{p}^{2}+m_{a}^{2}}$ is the energy of particle species $a$, where $m_{a}$ is current mass of $a$ th species. In the weak magnetic field, we do not consider the trivial setup in which the electric field is parallel to the direction of magnetic field, i.e., 
E $\| \mathbf{B}$ because there is no Lorentz force term to bend the trajectory of a charged particle. We take $\mathbf{E}=\left(E_{x}, E_{y}, 0\right)$ and $\mathbf{B}=(0,0, B)$ in the system so that the Hall effect and the Nernst effect can exist. The right side of Eq. (1) is the collision term or collision integral, which describes the rate of change of the single-particle distribution induced by scatterings. We assume the system is close to the local thermodynamic equilibrium, and introduce the commonly used relaxation time approximation (RTA). In the RTA, the collision term can be expressed as

$C\left[f_{a}\right] \simeq-\frac{\delta f_{a}}{\tau_{a}}$.

Here, $\tau_{a}$ is the relaxation time of species $a$ which describes how fast the system reaches the equilibrium again. And $\delta f_{a}$ is the infinitesimal change in distribution function due to external disturbance,

$\delta f_{a}=f_{a}-\bar{f}_{a}$,

with $\bar{f}_{a}$ being the local equilibrium distribution function of $a$ th species. In an isotropic medium or an anisotropic medium caused by initial spatial expansion, $\bar{f}_{a}$ in the local rest frame can be expressed as [71]

$\bar{f}_{a}\left(\mathbf{p}, \mu_{a}\right)=\left\{\begin{array}{l}\bar{f}_{a}^{0}=\frac{1}{e^{\left(\sqrt{\mathbf{p}^{2}+m_{a}^{2}}-\mu_{a}\right) \beta} \pm 1}, \quad(\xi=0) \\ \bar{f}_{a}^{\xi}=\frac{1}{e^{\left(\sqrt{\mathbf{p}^{2}+\xi(\mathbf{p} \cdot \mathbf{n})^{2}+m_{a}^{2}}-\mu_{a}\right) \beta} \pm 1}, \quad(\xi \neq 0)\end{array}\right.$

where $\beta=1 / T$ and $\mu_{a}$ denote inverse temperature and chemical potential of species $a$, respectively. \pm in Eq. (4) corresponds to fermions and bosons, separately. The anisotropic parameter $\xi$ in Eq. (4) is defined as [71]

$\xi=\frac{\left\langle\mathbf{p}_{\perp}^{2}\right\rangle}{2\left\langle p_{\|}^{2}\right\rangle}-1$,

where $p_{\|}=\mathbf{p} \cdot \mathbf{n}$ and $\mathbf{p}_{\perp}=\mathbf{p}-\mathbf{n}(\mathbf{p} \cdot \mathbf{n})$ are the momentum components which are parallel and perpendicular to the momentum anisotropy direction (n), respectively. $\mathbf{p}=(|\mathbf{p}| \sin \theta \cos \phi,|\mathbf{p}| \sin \theta \sin \phi,|\mathbf{p}| \cos \theta)$, where we use a notation $|\mathbf{p}| \equiv p$ for convenience. $\mathbf{n}=(\sin \alpha, 0, \cos \alpha)$, where $\alpha$ is the angle between $z$ direction and $\mathbf{n}$ direction. Accordingly, $(\mathbf{p} \cdot \mathbf{n})^{2}=p^{2}(\sin \theta \cos \phi \sin \alpha+\cos \theta \cos \alpha)^{2}=$ $p^{2} c(\theta, \phi, \alpha)$. In this work, we consider the anisotropy direction is along the beam direction, so $\alpha$ is fixed as $\pi / 2$, $\mathbf{n}=(1,0,0)$. Note that $\xi>0$ stands for a contraction of distribution function along $\mathbf{n}$ direction whereas $-1<\xi<0$ represents the stretching of distribution function along $\mathbf{n}$ direction. As mentioned in Section. 1, for the anisotropic QGP induced by the initial spatial expansion, $\xi$ is always larger than zero. In the weak $\xi$ limit $(|\xi| \ll 1), \bar{f}_{a}^{\xi}$ can be expanded in Taylor series to leading order term of $\xi$,

$\bar{f}_{a}^{\xi}=\bar{f}_{a}^{0}-\frac{\xi \beta}{2 \epsilon_{a}} \bar{f}_{a}^{0}\left(1-\bar{f}_{a}^{0}\right)(\mathbf{p} \cdot \mathbf{n})^{2}$.
Considering the distribution function and chemical potential are time independent and space-time independent, respectively, Eq. (1) can be rewritten as

$$
\begin{gathered}
{\left[v_{a}-e_{a} B\left(v_{x} \frac{\partial}{\partial p_{y}}-v_{y} \frac{\partial}{\partial p_{x}}\right)\right] f_{a}} \\
=v_{a} \bar{f}_{a}-e_{a} E_{x} \frac{\partial}{\partial p_{x}} \bar{f}_{a} \\
\quad-e_{a} E_{y} \frac{\partial}{\partial p_{y}} \bar{f}_{a}-\left(\mathbf{v}_{a} \cdot \nabla \bar{f}_{a}\right),
\end{gathered}
$$

where $v_{a}=1 / \tau_{a}$ is the inverse relaxation time of $a$ th species. We further assume the solution of Eq. (7) in an anisotropic medium satisfies the following linear form,

$f_{a}^{\xi}=\bar{f}_{a}^{\xi}-\frac{1}{v_{a}} e \mathbf{E} \cdot \frac{\partial \bar{f}_{a}^{\xi}}{\partial \mathbf{p}}-\Xi \cdot \frac{\partial \bar{f}_{a}^{\xi}}{\partial \mathbf{p}}-\frac{1}{v_{a}} \mathbf{v} \cdot \nabla \bar{f}_{a}^{\xi}$,

with $\Xi$ being an unknown quantity related to magnetic field. Inserting Eq. (8) into Eq. (7) and assuming no temperature gradient exists along $z$-axis, we obtain

$$
\begin{aligned}
0= & v_{a} F_{a}\left(\Xi_{x} v_{x}+\Xi_{y} v_{y}\right) \\
& +\frac{\omega_{c, a} F_{a}}{v_{a}}\left(e E_{x} v_{y}-e E_{y} v_{x}\right) \\
& +\frac{\omega_{c, a}}{v_{a}} G_{a}\left(\nabla_{y} T v_{x}-\nabla_{x} T v_{y}\right) \\
& +\omega_{c, a} F_{a}\left(\Xi_{x} v_{y}-\Xi_{y} v_{x}\right)
\end{aligned}
$$

where $\omega_{c, a}=e_{a} B / \epsilon_{a}$ is the cyclotron frequency of species $a$. The expressions of $G_{a}$ and $F_{a}$ in Eq. (9) can read as

$$
\begin{aligned}
F_{a}= & \beta \bar{f}_{a}^{0}\left(1 \pm \bar{f}_{a}^{0}\right)(1+\xi c(\theta, \phi, \alpha)) \\
& -\frac{\xi \beta^{2} p^{2} c(\theta, \phi, \alpha)}{2 \epsilon_{a}} \bar{f}_{a}^{0}\left(1 \pm \bar{f}_{a}^{0}\right) \\
& \times\left(1-\bar{f}_{a}^{0} \pm \bar{f}_{a}^{0}+\frac{1}{\beta \epsilon_{a}}\right), \\
G_{a}= & \beta^{2}\left(\epsilon_{a}-\mu_{a}\right) \bar{f}_{a}^{0}\left(1 \pm \bar{f}_{a}^{0}\right) \\
& -\frac{\xi \beta^{3} p^{2} c(\theta, \phi, \alpha)}{2 \epsilon_{a}}\left(\epsilon_{a}-\mu_{a}\right) \bar{f}_{a}^{0}\left(1 \pm \bar{f}_{a}^{0}\right) \\
& \times\left(1-\bar{f}_{a}^{0} \pm \bar{f}_{a}^{0}-\frac{1}{\beta\left(\epsilon_{a}-\mu_{a}\right)}\right),
\end{aligned}
$$

Comparing the coefficients of $v_{x}$ and $v_{y}$, one gets

$$
\begin{aligned}
& v_{a} F_{a} \Xi_{x}-\omega_{c, a} \frac{e E_{y}}{v_{a}} F_{a}-\omega_{c, a} F_{a} \Xi_{y} \\
& +\frac{\omega_{c, a}}{v_{a}} G_{a} \nabla_{y} T=0, \\
& v_{a} F_{a} \Xi_{y}+\omega_{c, a} \frac{e E_{x}}{v_{a}} F_{a}+\omega_{c, a} F_{a} \Xi_{x} \\
& -\frac{\omega_{c, a}}{v_{a}} G_{a} \nabla_{x} T=0 .
\end{aligned}
$$


Solving Eqs. (12) and (13), we obtain

$$
\begin{aligned}
\Xi_{x}= & -\frac{\omega_{c, a}^{2} e E_{x}}{v_{a}\left(\omega_{c, a}^{2}+v_{a}^{2}\right)} \\
& +\frac{\omega_{c, a} e E_{y}}{\omega_{c, a}^{2}+v_{a}^{2}}-\frac{\omega_{c, a} G_{a}}{F_{a}\left(\omega_{c, a}+v_{a}^{2}\right)} \nabla_{y} T \\
& +\frac{\omega_{c, a}^{2} G_{a}}{v_{a} F_{a}\left(\omega_{c, a}^{2}+v_{a}^{2}\right)} \nabla_{x} T, \\
\Xi_{y}= & -\frac{\omega_{c, a} e E_{x}}{\omega_{c, a}+v_{a}^{2}} \\
& -\frac{\omega_{c, a}^{2} e E_{y}}{v_{a}\left(\omega_{c, a}^{2}+v_{a}^{2}\right)}+\frac{\omega_{c, a}^{2} G_{a}}{F_{a} v_{a}\left(\omega_{c, a}^{2}+v_{a}^{2}\right)} \nabla_{y} T \\
& +\frac{\omega_{c, a} G_{a}}{F_{a}\left(\omega_{c, a}^{2}+v_{a}^{2}\right)} \nabla_{x} T .
\end{aligned}
$$

Inserting Eqs. (14)-(15) to Eq. (8), we finally obtain the following perturbative term,

$$
\begin{aligned}
\delta f_{a}^{\xi}= & f_{a}^{\xi}-\bar{f}_{a}^{\xi}=\frac{\omega_{c, a} F_{a}}{\left(\omega_{c, a}^{2}+v_{a}^{2}\right)}\left(e E_{y} v_{x}-e E_{x} v_{y}\right) \\
& +\frac{v_{a} F_{a}}{\omega_{c, a}^{2}+v_{a}^{2}}\left(e E_{x} v_{x}+e E_{y} v_{y}\right) \\
& +\frac{\omega_{c, a} G_{a}}{\omega_{c, a}^{2}+v_{a}^{2}}\left(\nabla_{x} T v_{y}-\nabla_{y} T v_{x}\right) \\
& -\frac{v_{a} G_{a}}{\omega_{c, a}^{2}+v_{a}^{2}}\left(\nabla_{x} T v_{x}+\nabla_{y} T v_{y}\right) .
\end{aligned}
$$

In a conducting medium, the charge carriers moving along the direction of thermal current generated by the thermal gradient accumulate on the cold side and an electric field can be generated. This electric field in turn induces an electric current in the opposite direction to the thermal current, consequently, a net electric current may exist in the medium. When a magnetic field perpendicular to the thermal gradient (assuming the thermal gradient is along $x$ direction) is applied, the charge carriers moving along the direction of thermal gradient and along induced electric field can be deflected, a net electric current perpendicular to both the thermal gradient and magnetic field can also be generated. Hence, in the linear response theory, the general formula of electric current density $\left(\mathbf{J}_{a}\right)$ for species $a$ in response to electric field (E) and temperature gradient $(\nabla T)$ is given by [82-85]

$\mathbf{J}_{a}=e_{a} \int \frac{d^{3} \mathbf{p}}{(2 \pi)^{3}} \mathbf{v} \delta f_{a}=\hat{\sigma}_{a} \cdot \mathbf{E}+\hat{\alpha}_{a}(-\nabla T)$,

where $\hat{\sigma}_{a}$ and $\hat{\alpha}_{a}$ are the electrical conductivity tensors and the thermoelectric conductivity tensors for species $a$, respectively. And the electric current density can further decompose as $[86,87]$

$$
\begin{aligned}
J_{x, a}= & \sigma_{x x, a} E_{x}+\sigma_{x y, a} E_{y}+\alpha_{x x, a}\left(-\nabla_{x} T\right) \\
& +\alpha_{x y, a}\left(-\nabla_{y} T\right),
\end{aligned}
$$

$$
\begin{aligned}
J_{y, a}= & \sigma_{y y, a} E_{y}+\sigma_{y x, a} E_{x}+\alpha_{y y, a}\left(-\nabla_{y} T\right) \\
& +\alpha_{y x, a}\left(-\nabla_{x} T\right) .
\end{aligned}
$$

The first term in Eq. (18) (Eq. (19)) is the electric current due to the electric field along $x(y)$-axis induced by the more accumulating carriers on the cold side of medium, and the third term in Eq. (18) (Eq. (19)) is the thermal current due to the thermal gradient in $x(y)$-axis. The second term and fourth term in Eq. (18) (Eq. (19)) are deuterogenic terms due to the deflection of the first term and third term in Eq. (19) (Eq. (18)) by the magnetic field directed along $z$-axis.

In the steady state (i.e., putting $J_{x, a}=J_{y, a}=0$ ), to avoid complicating the unambiguous determination of the Seebeck coefficient and the Nernst signal, we assume the thermal gradient purely along $x$-axis, i.e. $\nabla_{x} T \neq 0$, $\nabla_{y} T=0$ (isothermal condition). Using the Onsager reciprocity relation of the thermoelectric and electric conductivity tensors in a magnetic field, $\sigma_{x x, a}\left(\alpha_{x x, a}\right)=\sigma_{y y, a}\left(\alpha_{y y, a}\right)$ and $\sigma_{x y, a}\left(\alpha_{x y, a}\right)=-\sigma_{y x, a}\left(-\alpha_{y x, a}\right)$, we finally derive the expressions of the Seebeck coefficient $\left(S_{x x, a}\right)$ and the Nernst signal $\left(N_{a}\right)$ for $a$ th species from Eqs. (18) and (19) [86-89]

$$
\begin{aligned}
S_{x x, a} & =\left.\frac{E_{x}}{\nabla_{x} T}\right|_{J_{x, a}=J_{y, a}=0} \\
& =\frac{\sigma_{x x, a} \alpha_{x x, a}+\sigma_{x y, a} \alpha_{x y, a}}{\sigma_{x x, a}^{2}+\sigma_{x y, a}^{2}}, \\
N_{a} & =\left.\frac{E_{y}}{\nabla_{x} T}\right|_{J_{x, a}=J_{y, a}=0} \\
& =\frac{\sigma_{x y, a} \alpha_{x x, a}-\sigma_{x x, a} \alpha_{x y, a}}{\sigma_{x x, a}^{2}+\sigma_{x y, a}^{2}} .
\end{aligned}
$$

Inserting Eq. (16) to Eq. (17) and using Eqs. (18) and (19), by intergrating over $\theta$ and $\phi$, we get the expressions of the electrical conductivity $\left(\sigma_{x x, a}\right)$ and the Hall conductivity $\left(\sigma_{x y, a}\right)$ of $a$ th species in an anisotropic medium, which can be presented in a matrix form

$$
\begin{aligned}
\left(\begin{array}{c}
\sigma_{x x, a} \\
\sigma_{x y, a}
\end{array}\right)= & d_{a} \frac{e^{2} q_{a}^{2} \beta}{6} \int \frac{d p}{\left(\pi^{2}\right)} \frac{p^{4}}{\epsilon_{a}^{2}} \frac{1}{\omega_{c, a}^{2}+\left(v_{a}^{\xi}\right)^{2}}\left(\begin{array}{c}
v_{a}^{\xi} \\
\omega_{c, a}
\end{array}\right) \\
& \bar{f}_{a}^{0}\left(1 \pm \bar{f}_{a}^{0}\right)\left(1+\frac{\xi}{3}\right) \\
& -d_{a} \frac{e^{2} q_{a}^{2} \beta^{2} \xi}{36} \int \frac{d p}{\pi^{2}} \frac{p^{6}}{\epsilon_{a}^{3}} \frac{1}{\omega_{c, a}^{2}+\left(v_{a}^{\xi}\right)^{2}} \\
& \times\left(\begin{array}{c}
v_{a}^{\xi} \\
\omega_{c, a}
\end{array}\right) f_{a}^{0}\left(1 \pm \bar{f}_{a}^{0}\right)\left(1-\bar{f}_{a}^{0} \pm \bar{f}_{a}^{0}+\frac{1}{\beta \epsilon_{a}}\right),
\end{aligned}
$$

where $d_{a}$ and $v_{a}^{\xi}$ are degeneracy factor and the $\xi$-dependent inverse relaxation time for particle species $a$, respectively. When $\xi=0$, Eq. (22) reduces to the standard form in the 
isotropic medium,

$$
\begin{aligned}
\left(\begin{array}{c}
\sigma_{x x, a} \\
\sigma_{x y, a}
\end{array}\right)= & d_{a} \frac{e^{2} q_{a}^{2} \beta}{3} \int \frac{d^{3} \mathbf{p}}{(2 \pi)^{3}} \frac{p^{2}}{\epsilon_{a}^{2}} \frac{1}{\omega_{c, a}^{2}+\left(v_{a}\right)^{2}}\left(\begin{array}{c}
v_{a} \\
\omega_{c, a}
\end{array}\right) \\
& \times \bar{f}_{a}^{0}\left(1 \pm \bar{f}_{a}^{0}\right) .
\end{aligned}
$$

Accordingly, the thermoelectric conductivity $\left(\alpha_{x x, a}\right)$ and the Hall-like thermoelectric conductivity $\left(\alpha_{x y, a}\right)$ of $a$ th species within the effect of momentum anisotropy also can read as

$$
\begin{aligned}
\left(\begin{array}{c}
\alpha_{x x, a} \\
\alpha_{x y, a}
\end{array}\right)= & d_{a} \frac{e q_{a} \beta^{2}}{6} \int \frac{d p}{\pi^{2}} \frac{p^{4}}{\epsilon_{a}^{2}} \frac{\epsilon_{a}-\mu_{a}}{\omega_{c, a}^{2}+\left(v_{a}^{\xi}\right)^{2}} \\
& \left(\begin{array}{c}
v_{a}^{\xi} \\
\omega_{c, a}
\end{array}\right) \bar{f}_{a}^{0}\left(1 \pm \bar{f}_{a}^{0}\right) \\
& -d_{a} \frac{e q_{a} \beta^{3} \xi}{36} \int \frac{d p}{\pi^{2}} \frac{p^{6}}{\epsilon_{a}^{3}} \frac{1}{\omega_{c, a}^{2}+\left(v_{a}^{\xi}\right)^{2}}\left(\begin{array}{c}
v_{a}^{\xi} \\
\omega_{c, a}
\end{array}\right) \\
& \times\left(\epsilon_{a}-\mu_{a}\right) \bar{f}_{a}^{0}\left(1 \pm \bar{f}_{a}^{0}\right) \\
& \times\left(1-\bar{f}_{a}^{0} \pm \bar{f}_{a}^{0}-\frac{1}{\beta\left(\epsilon_{a}-\mu_{a}\right)}\right) .
\end{aligned}
$$

In the isotropic medium, the matrix of the thermoelectric conductivity tensors can be simplified as

$$
\begin{aligned}
\left(\begin{array}{c}
\alpha_{x x, a} \\
\alpha_{x y, a}
\end{array}\right)= & d_{a} \frac{e q_{a} \beta^{2}}{3} \int \frac{d^{3} \mathbf{p}}{(2 \pi)^{3}} \frac{p^{2}}{\epsilon_{a}^{2}} \frac{\epsilon_{a}}{\omega_{c, a}^{2}+v_{a}^{2}}\left(\begin{array}{c}
v_{a} \\
\omega_{c, a}
\end{array}\right) \\
& \times \bar{f}_{a}^{0}\left(1 \pm \bar{f}_{a}^{0}\right)-d_{a} \frac{e q_{a} \beta^{2}}{3} \\
& \times \int \frac{d^{3} \mathbf{p}}{(2 \pi)^{3}} \frac{p^{2}}{\epsilon_{a}^{2}} \frac{\mu_{a}}{\omega_{c, a}^{2}+v_{a}^{2}}\left(\begin{array}{c}
v_{a} \\
\omega_{c, a}
\end{array}\right) \bar{f}_{a}^{0}\left(1 \pm \bar{f}_{a}^{0}\right) .
\end{aligned}
$$

Since the total Seebeck coefficient $\left(S_{x x}\right)$ and the total Nernst signal $(N)$ are the sum of contributions from different types of carriers weighted by the respective electrical conductivities [90-93], in the QGP with three-flavor (anti-)quarks ( $f=$ $u, d, s)$, the expressions of $S_{x x}$ and $N$ can be naturally written as,

$$
\begin{aligned}
S_{x x} & =\frac{\sum_{f}\left(S_{x x, q_{f}} \sigma_{x x, q_{f}}+S_{x x, \bar{q}_{f}} \sigma_{x x, \bar{q}_{f}}\right)}{\sum_{f}\left(\sigma_{x x, q_{f}}+\sigma_{x x, \bar{q}_{f}}\right)}, \\
N & =\frac{\sum_{f}\left(N_{q_{f}} \sigma_{x x, q_{f}}+N_{\bar{q}_{f}} \sigma_{x x, \bar{q}_{f}}\right)}{\sum_{f}\left(\sigma_{x x, q_{f}}+\sigma_{x x, \bar{q}_{f}}\right)} .
\end{aligned}
$$

The denominator in Eq. (26) denotes the total electrical conductivity $\left(\sigma_{x x}\right)$, accordingly other total conductivity tensors from different flavors (anti)-quarks also can be given as $\sigma_{x y}=$ $\sum_{f}\left(\sigma_{x y, q_{f}}+\sigma_{x y, \bar{q}_{f}}\right), \alpha_{x x}=\sum_{f}\left(\alpha_{x x, q_{f}}+\alpha_{x x, \bar{q}_{f}}\right), \alpha_{x y}=$ $\sum_{f}\left(\alpha_{x y, q_{f}}+\alpha_{x y, \bar{q}_{f}}\right)$. When the magnetic field is turned off, the Nernst effect is absent and only the Seebeck effect exists. At the same time, the expression of $S_{x x, a}$ reduces to $S_{x x, a}=\frac{\alpha_{x x, a}}{\sigma_{x x, a}}$. We note that the conductivity tensors are coupling-constant-dependent, however, the Seebeck coefficient and Nernst signal are unaffected by different coupling constants. It can be understood that the coupling constant term, which is embedded in the relaxation time, in the numerator of Eq. (26) is exactly cancelled by that in the denominator.

\section{Thermoelectric coefficients in an (an-)isotropic medium at strong magnetic field}

At strong magnetic field directed in $z$-axis within the regime $e B \gg T^{2}$, in the significant Landau quantization, if we still take electric field along $x$-direction, the electrical conductivity $\left(\sigma_{x x}\right)$ is zero in the one-loop calculation [94] and the Hall conductivity becomes $\sigma_{x y}=n_{e} / B$ ( $n_{e}$ is electron number density) [33]. Very recently, S. Lin and L. Yang have deduced the general formulas of $\sigma_{x x}$ and $\sigma_{x y}$ in chiral kinetic theory with full Landau level basis at the strong magnetic field along $z$-axis [95]. However, due to the lack of corresponding collision term, it still remains a great challenge to fully understand the flavor dynamics of a magnetized QCD plasma in the background of strong magnetic field perpendicular to electric field. Accordingly, we focus on a simply setup in which the direction of electric field is parallel to the direction of magnetic field. Therefore, the linear Boltzmann equation of the magnetic-field-dependent single particle distribution $f_{B, a}$ at an external electric field $\mathbf{E}=\left(0,0, E_{z}\right)$ in the RTA can be given as

$\frac{\partial f_{B, a}}{\partial t}+v_{z} \nabla_{z} f_{B, a}+e_{a} E_{z} \frac{\partial f_{B, a}}{\partial p_{z}}=-\frac{\delta f_{B, a}}{\tau_{B, a}}$,

where the correction term $\delta f_{B, a}$ can be written as

$\delta f_{B, a}=-\tau_{B, a}\left[v_{z} \nabla_{z} \bar{f}_{B, a}+e q_{a} E_{z} \frac{\partial \bar{f}_{B, a}}{\partial p_{z}}\right]$.

Due to the motion of (anti-)quarks in the Landau quantization is mainly restricted to the direction of magnetic field, the equilibrium distribution function of charge particle $\bar{f}_{B, a}$ in an isotropic medium and in an anisotropic medium induced by strong magnetic field can read as

$\bar{f}_{B, a}\left(p_{z}, \mu_{a}\right)=\left\{\begin{array}{l}\bar{f}_{B, a}^{0}=\frac{1}{e^{\left(\epsilon_{a, l}-\mu_{a}\right) \beta} \pm 1},\left(\xi^{\prime}=0\right), \\ \bar{f}_{B, a}^{\xi^{\prime}}=\frac{1}{e^{\left(\sqrt{\epsilon_{a, l}^{2}+\xi^{\prime}\left(\mathbf{p} \cdot \mathbf{n}^{\prime}\right)^{2}}-\mu_{a}\right) \beta} \pm 1},\left(\xi^{\prime} \neq 0\right) .\end{array}\right.$

Here we assume that the direction of momentum anisotropy $\mathbf{n}^{\prime}$ is directed in the direction of magnetic field, and $\mathbf{p} \approx$ $\left(0,0, p_{z}\right)$, as Refs. [77,78]. Unlike the anisotropic parameter $\xi$, the anisotropic parameter induced by strong magnetic field $\left(\xi^{\prime}\right)$ is always negative because the momentum component along the direction of momentum anisotropy (viz, along the direction of magnetic field) is dominant as compared to that along other directions. In the small $\xi^{\prime}$ limit $\left(\left|\xi^{\prime}\right| \ll 1\right), \bar{f}_{B, a}^{\xi^{\prime}}$ 
also can be expanded to leading order of $\xi^{\prime}$,

$$
\bar{f}_{B, a}^{\xi^{\prime}}=\bar{f}_{B, a}^{0}-\frac{\xi^{\prime} \beta p_{z}^{2}}{2 \epsilon_{a, l}} \bar{f}_{B, a}^{0}\left(1 \pm \bar{f}_{B, a}^{0}\right) .
$$

The phase space integration for $a$ th charged particle due to the dimensional reduction of motion in Landau quantization is modified to $\int \frac{d^{3} \mathbf{p}}{(2 \pi)^{3}}=\sum_{l=0}^{\infty} \frac{\left|q_{a} e B\right|}{2 \pi} \int_{-\infty}^{+\infty} \frac{d p_{z}}{2 \pi}$, where $e B /(2 \pi)$ is the density of states in two spatial directions perpendicular to the direction of magnetic field $[32,96]$. In the linear response theory, the longitudinal electric current density of species $a$ can be written as

$$
\begin{aligned}
J_{z, a} & =\sum_{l} g_{a, l} q_{a} e \frac{\left|q_{a} e B\right|}{2 \pi} \int_{-\infty}^{+\infty} \frac{d p_{z}}{2 \pi} v_{z} \delta f_{B, a} \\
& =\sigma_{z z, a} E_{z}+\alpha_{z z, a}\left(-\nabla_{z} T\right),
\end{aligned}
$$

with $g_{a, l}$ being the degeneracy factor of $a$ th species. For quarks and anti-quarks, $g_{a, l}=\sum_{f}\left(2-\delta_{0 l}\right) N_{c}$, in which $\left(2-\delta_{0 l}\right)$ and $N_{c}$ are spin degeneracy factor of the Landau levels and the number of quark colors, respectively. Since the direction of temperature gradient is parallel to the direction of magnetic field in the strongly magnetized medium, the Nernst effect vanishes. Setting $J_{z z, a}=0$, the longitudinal Seebeck coefficient of $a$ th species, $S_{z z, a}$, can be expressed as $S_{z z, a}=\frac{E_{z}}{\nabla_{z} T}=\frac{\alpha_{z z, a}}{\sigma_{z z} a}$, where $\sigma_{z z, a}\left(\alpha_{z z, a}\right)$ is the longitudinal electrical (thermoelectric) conductivity of $a$ th species. Using Eqs. (28)-(31) the formulas of $\sigma_{z z, a}$ and $\alpha_{z z, a}$ in the (an)isotropic medium can be respectively derived as,

$$
\begin{aligned}
\sigma_{z z, a}= & -e^{2} q_{a}^{2} \frac{\left|q_{a} e B\right| \beta^{2} \xi^{\prime}}{2 \pi} \sum_{l} g_{a, l} \\
& \times \int_{-\infty}^{+\infty} \frac{d p_{z}}{2 \pi} \frac{p_{z}^{4}}{2 \epsilon_{a, l}^{3}} \tau_{B, a}^{\xi^{\prime}} \bar{f}_{B, a}^{0}\left(1 \pm \bar{f}_{B, a}^{0}\right) \\
& \left(1-2 \bar{f}_{B, a}^{0}+\frac{1}{\beta \epsilon_{a, l}}\right) \\
& +e^{2} q_{a}^{2} \frac{\left|q_{a} e B\right| \beta}{2 \pi} \sum_{l} g_{a, l} \\
& \times \int_{-\infty}^{+\infty} \frac{d p_{z}}{2 \pi} \frac{p_{z}^{2}}{\epsilon_{a, l}^{2}} \tau_{B, a}^{\xi^{\prime}} \bar{f}_{a, B}^{0} \\
& \left(1 \pm \bar{f}_{B, a}^{0}\right)\left(1+\xi^{\prime}\right), \quad\left(\text { for } \xi^{\prime} \neq 0\right) ; \\
= & e^{2} q_{a}^{2} \frac{\left|q_{a} e B\right| \beta}{2 \pi} \sum_{l} g_{a, l} \\
& \times \int_{-\infty}^{+\infty} \frac{d p_{z}}{2 \pi} \frac{p_{z}^{2}}{\epsilon_{a, l}^{2}} \tau_{B, a} \bar{f}_{B, a}^{0}\left(1 \pm \bar{f}_{B, a}^{0}\right), \quad\left(\text { for } \xi^{\prime}=0\right) ;
\end{aligned}
$$$$
\begin{aligned}
\alpha_{z z, a}= & -e q_{a} \frac{\left|q_{a} e B\right| \beta^{3} \xi^{\prime}}{2 \pi} \sum_{l} g_{a, l} \\
& \times \int_{-\infty}^{+\infty} \frac{d p_{z}}{2 \pi} \frac{p_{z}^{4}}{2 \epsilon_{a, l}^{3}} \tau_{B, a}^{\xi^{\prime}}\left(\epsilon_{a, l}-\mu_{a}\right) \bar{f}_{B, a}^{0}\left(1 \pm \bar{f}_{a}^{0}\right)
\end{aligned}
$$

$$
\begin{aligned}
& \times\left(1-2 \bar{f}_{B, a}^{0}-\frac{1}{\beta\left(\epsilon_{a, l}-\mu_{a}\right)}\right) \\
& +e q_{a} \frac{\left|q_{a} e B\right| \beta^{2}}{2 \pi} \sum_{l} g_{a, l} \\
& \times \int_{-\infty}^{+\infty} \frac{d p_{z}}{2 \pi} \frac{p_{z}^{2}}{\epsilon_{a, l}^{2}} \tau_{B, a}^{\xi^{\prime}}\left(\epsilon_{a, l}-\mu_{a}\right) \bar{f}_{B, a}^{0} \\
& \times\left(1 \pm \bar{f}_{B, a}^{0}\right), \quad\left(\text { for } \xi^{\prime} \neq 0\right) ; \\
& =e q_{a} \frac{\left|q_{a} e B\right| \beta^{2}}{2 \pi} \sum_{l} g_{a, l} \\
& \times \int_{-\infty}^{+\infty} \frac{d p_{z}}{2 \pi} \frac{p_{z}^{2}}{\epsilon_{a, l}^{2}} \tau_{B, a}\left(\epsilon_{a, l}-\mu_{a}\right) \bar{f}_{B, a}^{0} \\
& \times\left(1 \pm \bar{f}_{B, a}^{0}\right), \quad\left(\text { for } \xi^{\prime}=0\right) .
\end{aligned}
$$

Here $\tau_{B, a}\left(\tau_{B, a}^{\xi^{\prime}}\right)$ is the magnetic-field-dependent relaxation time of species $a$ in the isotropic (anisotropic) medium. Finally, the total longitudinal Seebeck coefficient in the QGP with three-flavor can be given as

$$
\begin{aligned}
S_{z z} & =\frac{\sum_{f}\left(S_{z z, q_{f}} \sigma_{z z, q_{f}}+S_{z z, \bar{q}_{f}} \sigma_{z z, \bar{q}_{f}}\right)}{\sum_{f}\left(\sigma_{z z, q_{f}}+\sigma_{z z, \bar{q}_{f}}\right)} \\
= & \frac{\sum_{f}\left(\alpha_{z z, q_{f}}+\alpha_{z z, \bar{q}_{f}}\right)}{\sum_{f}\left(\sigma_{z z, q_{f}}+\sigma_{z z, \bar{q}_{f}}\right)}=\frac{\alpha_{z z}}{\sigma_{z z}},
\end{aligned}
$$

with $\alpha_{z z}\left(\sigma_{z z}\right)$ being the total longitudinal thermoelectric (electrical) conductivity.

\section{Thermal relaxation time}

In this work, the relaxation time is a vital dynamic input for the calculation of thermoelectric coefficients in the QGP. The computation of relaxation time inevitably involves the choice of effective running coupling constant, which can control the behavior of transport parameters critically. The effect of momentum anisotropy also can enter the effective coupling constant through the calculation of Debye mass using the anisotropic distribution functions. Conventionally, the Debye screening mass is obtained by the static limit of the gluon self-energy in Hard Thermal Loop (HTL) theory [97]. In this work, we use a parallel approach, i.e., the semiclassical transport theory [98-100] to get it. Furthermore, as the Debye screening mass manifests itself in the collective oscillation of the medium through the dispersion relation [101], accordingly it can be affected by the Landau quantization. As mentioned in Sect. 1, the scattering processes are significantly different at weak and strong magnetic fields, therefore we split this section into two separate parts: (A) without Landau quantization, and (B) with Landau quantization. The effects of momentum anisotropy induced by initial spatial expansion and strong magnetic field also straightly 
enter into the relaxation time and the Debye mass through replacing the isotropic distribution functions in associated expressions with the momentum anisotropic counterparts.

\subsection{Without Landau quantization}

At weak magnetic field within the regime $g^{2} T^{2} \gg e B$, the motions of particles in the QGP are not affected by the magnetic field through the Landau quantization. The Debye mass $\left(m_{D}\right)$ in the isotropic medium for vanishing magnetic field can be expressed as [42]

$m_{D}^{2}=-4 \pi \alpha_{s} \int \frac{d^{3} \mathbf{p}}{(2 \pi)^{3}}\left[2 N_{c} \frac{d \bar{f}_{g}^{0}}{d p}+\sum_{f} \sum_{i=q_{f}, \bar{q}_{f}} \frac{d \bar{f}_{i}^{0}}{d p}\right]$,

where $\mu_{q}\left(\mu_{\bar{q}}\right)$ is quark (anti-quark) chemical potential and $\mu_{\bar{q}}=-\mu_{q}$. When $\mu_{q} / T \lesssim 1, m_{D}^{2}=4 \pi \alpha_{s} T^{2}\left[\left(\frac{N_{c}}{3}+\frac{N_{f}}{6}\right)\right.$ $\left.+\left(\frac{\mu_{q}}{T}\right)^{2} \frac{N_{f}}{2 \pi^{2}}\right]$ for the massless case, where $N_{f}$ represents the number of quark flavors. This result is equal to the leadingorder result in HTL approximation [102-107]. In the momentum anisotropic medium induced by initial spatial expansion, the Debye mass needs to be modified by roughly replacing the isotropic distribution function with the anisotropic distribution function, which can be written as

$\left(m_{D}^{\xi}\right)^{2}=4 \pi \alpha_{s} \int \frac{d^{3} \mathbf{p}}{(2 \pi)^{3}}\left[2 N_{c} F_{g}+N_{f}\left(F_{q}+F_{\bar{q}}\right)\right]$.

In an anisotropic medium induced by initial spatial expansion, the $\xi$-dependent effective coupling constant can be defined as $\alpha_{e f f}=\frac{\left(m_{D}\right)^{2}}{\left(m_{D}^{\xi}\right)^{2}} \alpha_{S}$, where $\alpha_{S}=\frac{6 \pi}{\left(33-2 N_{f}\right) \ln \left(\frac{2 \pi T}{\Lambda_{\bar{M} S}}\right)}$ is the one-loop running coupling at $\Lambda_{\bar{M} S}=176 \mathrm{MeV}$ for $N_{f}=3$ [108]. For the elastic process $a_{1}\left(P_{1}\right)+a_{2}\left(P_{2}\right) \rightarrow$ $a_{3}\left(P_{3}\right)+a_{4}\left(P_{4}\right)\left(P_{i=1,2,3,4}\right.$ is the four-momentum of $i$ th particle), the inverse relaxation time of species $a_{1}, 1 / \tau_{1}$, can be given as (see Appendix A for the detailed derivation)

$\tau_{1}^{-1}=\sum_{\text {pro }} \frac{d_{2}}{\delta_{34}+1} \int \frac{d^{3} \mathbf{p}_{2}}{(2 \pi)^{3}} \bar{f}_{2}\left(1 \pm \bar{f}_{4}\right) \int d t \frac{d \sigma^{\text {pro }}}{d t} \frac{2 t u}{s^{2}}$

where $s, t, u$ are Mandelstam variables, and $\frac{d \sigma^{\text {pro }}}{d t}$ is the differential cross section for a specific scattering process. In our work only the elastic scatterings, viz, (1) $g q \rightarrow g q$, (2) $q q \rightarrow q q$, (3) $q \bar{q} \rightarrow q \bar{q},(4) q q^{\prime} \rightarrow q q^{\prime},(5) q \bar{q}^{\prime} \rightarrow q \bar{q}^{\prime}$ are considered. We reasonably discard the inelastic processes like $q \bar{q} \rightarrow g g$ due to their small contributions. The differential cross sections in the leading order perturbative QCD calculation for the massless case can be found in Refs. $[109,110]$. Finally, the momentum-averaged thermal relaxation time of (anti-)quarks in the isotropic medium can be written as

$$
\begin{aligned}
& v_{q(\bar{q})}=\tau_{q(\bar{q})}^{-1}=d_{g} \int \frac{d^{3} \mathbf{p}}{(2 \pi)^{3}} \bar{f}_{g}^{0}(\mathbf{p})\left(1+\bar{f}_{g}^{0}(\mathbf{p})\right) \\
& \times \frac{1}{4} \frac{g^{4}}{\pi\left\langle s_{q(\bar{q}) g}\right\rangle}\left[\ln \frac{\left\langle s_{q(\bar{q}) g}\right\rangle}{\mu_{D}^{2}}-\frac{139}{108}\right] \\
& +d_{q(\bar{q})} \int \frac{d^{3} \mathbf{p}}{(2 \pi)^{3}} \bar{f}_{q(\bar{q})}^{0}\left(\mathbf{p}, \mu_{q(\bar{q})}\right) \\
& \left(1-\bar{f}_{q(\bar{q})}^{0}\left(\mathbf{p}, \mu_{q(\bar{q})}\right)\right) \\
& \times \frac{1}{9} \frac{g^{4}}{\pi\left\langle s_{q q(\bar{q} \bar{q})}\right\rangle}\left[\ln \frac{\left\langle s_{q q(\bar{q} \bar{q})}\right\rangle}{\mu_{D}^{2}}-\frac{152}{96}\right] \\
& +d_{\bar{q}(q)} \int \frac{d^{3} \mathbf{p}}{(2 \pi)^{3}} \bar{f}_{\bar{q}(q)}^{0}\left(\mathbf{p}, \mu_{\bar{q}(q)}\right) \\
& \left(1-\bar{f}_{\bar{q}(q)}^{0}\left(\mathbf{p}, \mu_{\bar{q}(q)}\right)\right) \\
& \times \frac{1}{9} \frac{g^{4}}{\pi\left\langle s_{q \bar{q}}\right\rangle}\left[\ln \frac{\left\langle s_{q \bar{q}}\right\rangle}{\mu_{D}^{2}}-\frac{147}{120}\right] \\
& +d_{q^{\prime}\left(\overline{q^{\prime}}\right)} \int \frac{d^{3} \mathbf{p}}{(2 \pi)^{3}} \bar{f}_{q^{\prime}\left(\overline{q^{\prime}}\right)}^{0}\left(\mathbf{p}, \mu_{q(\bar{q})}\right) \\
& \left(1-\bar{f}_{q^{\prime}\left(\overline{q^{\prime}}\right)}^{0}\left(\mathbf{p}, \mu_{q(\bar{q})}\right)\right) \\
& \times \frac{1}{9} \frac{g^{4}}{\pi\left\langle s_{q q(\bar{q} \bar{q})}\right\rangle}\left[\ln \frac{\left\langle s_{q q(\bar{q} \bar{q})}\right\rangle}{\mu_{D}^{2}}-\frac{17}{12}\right] \\
& +d_{\bar{q}^{\prime}\left(q^{\prime}\right)} \int \frac{d^{3} \mathbf{p}}{(2 \pi)^{3}} \bar{f}_{\bar{q}^{\prime}\left(q^{\prime}\right)}^{0}\left(\mathbf{p}, \mu_{\bar{q}(q)}\right) \\
& \left(1-\bar{f}_{\bar{q}^{\prime}\left(q^{\prime}\right)}^{0}\left(\mathbf{p}, \mu_{\bar{q}(q)}\right)\right) \\
& \times \frac{1}{9} \frac{g^{4}}{\pi\left\langle s_{q \bar{q}}\right\rangle}\left[\ln \frac{\left\langle s_{q \bar{q}}\right\rangle}{\mu_{D}^{2}}-\frac{17}{12}\right],
\end{aligned}
$$

where $\left\langle s_{i j}\right\rangle=2\left\langle p_{i}\right\rangle\left\langle p_{j}\right\rangle$ and $\left\langle p_{i}\right\rangle=\frac{\int \frac{d^{3} \mathbf{p}_{i}}{(2 \pi)^{3}}\left|\mathbf{p}_{i}\right| \bar{f}_{i}^{0}}{\int \frac{d^{3} \mathbf{p}}{(2 \pi)^{3}} \bar{f}_{i}^{0}}$ is the thermal average value of $p_{i}$. And in Eq. (40) the degeneracy factors of gluons and (anti-)quarks are $d_{g}=2_{\text {spin }} \times\left(N_{c}^{2}-1\right)$ and $d_{q(\bar{q})}=2_{\text {spin }} \times N_{c} \times N_{f}$, respectively. $q^{\prime}\left(\bar{q}^{\prime}\right)$ denotes incoming (anti-)quark, which is different to another incoming (anti)quark in flavor type. $\mu_{D}^{2}=g^{2} T^{2}$ denotes infrared regulator. In the anisotropic medium induced by initial spatial expansion, the $\xi$-dependent inverse relaxtion time of (anti-)quark, $v_{q(\bar{q})}^{\xi}$, can be obtained by substituting $\bar{f}_{i}^{0}$ and $\alpha_{s}$ in Eq. (40) with $\bar{f}_{i}^{\xi}$ and $\alpha_{e f f}$, respectively. Consequently, the thermal average of $p_{g}$ as well as $p_{q(\bar{q})}$ with anisotropic momentum distribution can be rewritten as

$\langle p\rangle_{i=g, q, \bar{q}}^{\xi}=T \frac{\operatorname{Li}_{4}\left(-e^{-\beta \mu_{i}}\right)}{\operatorname{Li}_{3}\left(-e^{-\beta \mu_{i}}\right)} \frac{4 \xi-6}{\xi-2}$,

with $\mathrm{Li}_{n}(z)$ being the PolyLog function. 


\subsection{With Landau quantization}

In the presence of strong magnetic field, only fermionic part of the Debye mass is affected by the Landau quantization, Eq. (37) can be modified as

$$
\begin{aligned}
m_{D, B}^{2}= & -4 \pi \alpha_{s, B}\left[\int \frac{d^{3} \mathbf{p}}{(2 \pi)^{3}} 2 N_{c} \frac{d}{d p} \bar{f}_{g}^{0}+\frac{1}{2} \sum_{f} \frac{\left|q_{f} e B\right|}{2 \pi}\right. \\
& \left.\times \sum_{l=0}^{\infty}\left(2-\delta_{0 l}\right) \int_{-\infty}^{+\infty} \frac{d p_{z}}{2 \pi} \sum_{i=q_{f}, \bar{q}_{f}} \frac{d \bar{f}_{B, i}^{0}}{d p}\right],
\end{aligned}
$$

where a QCD factor as $\frac{1}{2}$ is considered. In the regime of $T^{2} \ll e B$ within the LLL approximation, the running coupling constant mainly depends on magnetic field, which is given as $[16,79]$

$$
\begin{aligned}
\alpha_{s, B}^{-1}(B)= & \alpha_{s}^{0}\left(\mu_{0}\right)^{-1}+\frac{11 N_{c}}{12 \pi} \ln \left(\frac{\Lambda_{Q C D}^{2}+M_{B}^{2}}{\mu_{0}^{2}}\right) \\
& +\frac{1}{3 \pi} \sum_{f} \frac{\left|q_{f} e B\right|}{\sigma},
\end{aligned}
$$

where $\alpha_{s}^{0}\left(\mu_{0}\right)=\frac{12 \pi}{11 N_{c} \ln \left(\frac{\mu_{0}^{2}+M_{B}^{2}}{\Lambda_{V}^{2}}\right)}, M_{B}=1 \mathrm{GeV}$ and $\sigma=$ $0.18 \mathrm{GeV}^{2}$ are infrared mass and the string tension, respectively. In Refs. [16], $\Lambda_{V}$ and $\mu_{0}$ are taken as 0.385 and 1.1 $\mathrm{GeV}$, respectively. In the LLL approximation with the hierarchy of scale $e B \gg T^{2}$, Eq. (42) for the massless case reduces to

$$
\begin{aligned}
m_{D, B}^{2}= & 4 \pi \alpha_{s, B} T^{2} \frac{N_{c}}{3}+4 \pi \alpha_{s, B} \sum_{f} \frac{\left|q_{f} e B\right|}{4 \pi^{2}} \sum_{l=0}^{\infty}\left(2-\delta_{0 l}\right) \\
& \times\left[\frac{1}{e^{\left(\sqrt{2 l\left|q_{f} e B\right|}+\mu_{q}\right) / T}+1}+\frac{1}{e^{\left(\sqrt{2 l\left|q_{f} e B\right|}-\mu_{q}\right) / T}+1}\right] \\
= & 4 \pi \alpha_{s, B}\left[T^{2} \frac{N_{c}}{3}+\sum_{f} \frac{\left|q_{f} e B\right|}{4 \pi^{2}}\right] \\
\approx & \alpha_{s, B} \sum_{f} \frac{\left|q_{f} e B\right|}{\pi},
\end{aligned}
$$

which is consistent with the one-loop calculation in the presence of strong magnetic field $[41,48,111]$. Replacing $\bar{f}_{B, i}^{0}$ with $\bar{f}_{B, i}^{\xi^{\prime}}$ in Eq. (42), we can get the following $\xi^{\prime}$-dependent Debye mass $m_{D, B}^{\xi^{\prime}}$ in an anisotropic medium induced by strong magnetic field

$$
\begin{aligned}
\left(m_{D, B}^{\xi^{\prime}}\right)^{2}= & 4 \pi \alpha_{s, B} \sum_{f} \frac{\left|q_{f} e B\right|}{4 T \pi} \int_{-\infty}^{+\infty} \sum_{l=0}^{\infty} \frac{d p_{z}}{2 \pi}\left(2-\delta_{0 l}\right) \\
& \times \sum_{i=q, \bar{q}} H_{i},
\end{aligned}
$$

where $H_{i}=\bar{f}_{B, i}^{0}\left(1-\bar{f}_{B, i}^{0}\right)\left(1+\xi^{\prime}\right)-\frac{\xi^{\prime} \beta p_{z}^{2}}{2 \epsilon_{i, l}} \bar{f}_{B, i}^{0}\left(1-\bar{f}_{B, i}^{0}\right)(1-$ $2 \bar{f}_{B, i}^{0}+\frac{1}{\beta \epsilon_{i, l}}$. The effective coupling constant associated with momentum anisotropy induced by strong magnetic field is also defined as $\alpha_{e f f, B}=\left(m_{D, B}^{\xi^{\prime}}\right)^{2} \alpha_{S, B} / m_{D, B}^{2}$. In the strong magnetic field within the LLL approximation, except for the usual elastic $2 \rightarrow 2$ processes, the quark-antiquark pair to gluon $2 \rightarrow 1$ process and vice versa are also allowed, which is kinetically forbidden for weak or zero magnetic field case because two massive particle cannot become a massless particle. In the strong magnetic field, due to the spatial dimensional mixmatch between (anti-)quarks and gluons, the transverse momentum component of the gluon acts as " the gluon mass", so that the gluon can be generated by two massive particles and vice versa. And in the hierarchy $\alpha_{s, B} e B \ll T^{2} \ll e B$, when $m_{q}^{2} \gg \alpha_{s, B} e B$, the $2 \rightarrow 2$ processes are subleading compared to the $2 \rightarrow 1$ process because the typical scale of collision rate for $2 \rightarrow 2$ processes $\propto \alpha_{s, B}^{2}$ is parametrically smaller than the typical scale of $2 \rightarrow 1$ process $\propto \alpha_{s, B}$ (more details see Ref. [41]). However, when $m_{q}^{2} \ll \alpha_{s, B} e B$, the quark-antiquark $t$-channel scattering process and the $2 \rightarrow 1$ process are same order of running constant $\alpha_{s, B}$. Therefore, in present work, the collision terms with respect to two scattering processes, viz, quark-antiquark to gluon $q(P)+\bar{q}\left(P^{\prime}\right) \rightarrow g(K)$ and quark-antiquark $t$-channel scattering $q(P)+\bar{q}\left(P^{\prime \prime}\right) \rightarrow$ $q\left(P^{\prime}\right)+\bar{q}\left(P^{\prime \prime \prime}\right)$ are considered under the restrictive hierarchy of scales $m_{q}^{2} \ll \alpha_{s, B} e B \ll T^{2} \ll e B$. Following Ref. [41], we generalize the computation of the relaxation time of $f$ th (anti-)quarks for $2 \rightarrow 1$ process to the case of finite chemical potential $\left(\mu_{q} \neq 0\right)$ and anisotropic medium $\left(\xi^{\prime} \neq 0\right)$,

$$
\begin{aligned}
& \left.\frac{1}{\tau_{B, q(\bar{q})}^{\xi^{\prime}}}\right|_{2 \rightarrow 1}\left(T, p_{z}, \mu_{q}, l=0\right) \\
& =\frac{\alpha_{e f f, B} C_{R} m_{f}^{2}}{\epsilon_{f, 0}\left(1-\bar{f}_{B, q(\bar{q})}^{\xi^{\prime}}\left(p_{z}, \mu_{q(\bar{q})}\right)\right)} \\
& \quad \times \int_{-\infty}^{+\infty} \frac{d p_{z}^{\prime}}{\epsilon_{f, 0}^{\prime}} \bar{f}_{B, \bar{q}(q)}^{\xi^{\prime}}\left(p_{z}^{\prime}, \mu_{\bar{q}(q)}\right)\left(1+\bar{f}_{g}(k)\right) .
\end{aligned}
$$

Here $C_{R}=\frac{N_{c}^{2}-1}{2 N_{c}}$ is the Casimir factor. $f_{g}(k)=\frac{1}{e^{k / T}-1}$ with $k=|\mathbf{k}|=\epsilon_{f, 0}+\epsilon_{f, 0}^{\prime}$, where $\epsilon_{f, 0}=\sqrt{p_{z}^{2}+m_{f}^{2}}$ and $\epsilon_{f, 0}^{\prime}=$ $\sqrt{p_{z}^{\prime 2}+m_{f}^{2}}$. Furthermore, at nonzero $\mu_{q}$, the relaxation time of $f$ th (anti-)quarks for $2 \rightarrow 2$ process in an anisotropic medium is given as (details in Appendix B)

$$
\begin{aligned}
& \left.\frac{1}{\tau_{B, q(\bar{q})}^{\xi^{\prime}}}\right|_{2 \rightarrow 2}\left(T, p_{z}, \mu_{q}, l=0\right) \\
& =\alpha_{e f f, B} \frac{m_{f}^{2}}{\epsilon_{f, 0}}\left(1-\bar{f}_{B, \bar{q}(q)}^{\xi^{\prime}}\left(p_{z}, \mu_{\bar{q}(q)}\right)\right) .
\end{aligned}
$$


We can observe that the inverse relaxation times for $2 \rightarrow 1$ and $2 \rightarrow 2$ processes are of the same order of $\alpha_{e f f, B}$. Currently, the relaxation time of (anti-)quarks for $2 \rightarrow 1$ process beyond the LLL approximation at zero chemical potential has been studied in a more realistic regime $e B \gg g^{2} T^{2}$ $[33,44,52]$. Similarly, we extend it to the case of $\mu_{q} \neq 0$ and $\xi^{\prime} \neq 0$,

$$
\begin{aligned}
& \left.\frac{1}{\tau_{B, q(\bar{q})}^{\xi^{\prime}}}\right|_{2 \rightarrow 1}\left(T, p_{z}, \mu_{q}, l\right) \\
& =\frac{1}{4 \epsilon_{f, l}\left(1-\bar{f}_{B, q(\bar{q})}^{\xi^{\prime}}\left(p_{z}, \mu_{q(\bar{q})}, l\right)\right)} \\
& \quad \times \sum_{l^{\prime} \geq l}^{\infty} \int_{-\infty}^{+\infty} \frac{d p_{z}^{\prime}}{2 \pi} \frac{1}{2 \epsilon_{f, l^{\prime}}^{\prime}} \bar{f}_{B, \bar{q}(q)}^{\xi^{\prime}}\left(p_{z}^{\prime}, \mu_{\bar{q}(q)}, l^{\prime}\right) \\
& \quad \times\left(1+\bar{f}_{g}^{\xi^{\prime}}(k)\right) X\left(l, l^{\prime}, H\right) .
\end{aligned}
$$

Here $H$ is defined as $H=\frac{\left(\epsilon_{q, l}+\epsilon_{\bar{q}, l^{\prime}}\right)^{2}-\left(p_{z}+p_{z}^{\prime}\right)^{2}}{2 e\left|q_{f} B\right|}[33,44]$ and $X\left(l, l^{\prime}, H\right)$ can read as

$$
\begin{aligned}
X\left(l, l^{\prime}, H\right)= & 4 \pi \alpha_{e f f, B} C_{R} \frac{l !}{l^{\prime} !} e^{-H} H^{l^{\prime}-l}\left(\left[4 m_{f}^{2}\right.\right. \\
& \left.-4\left|q_{f} e B\right|\left(l+l^{\prime}-H\right) \frac{1}{H}\left(l+l^{\prime}\right)\right] F\left(l, l^{\prime}, H\right) \\
& \left.+16\left|q_{f} e B\right| l^{\prime}\left(l+l^{\prime}\right) \frac{1}{H} L_{l}^{l^{\prime}-l}(H) L_{l-1}^{l^{\prime}-l}(H)\right) .
\end{aligned}
$$

For $l>0, F\left(l, l^{\prime}, H\right)=\left[L_{l}^{l^{\prime}-l}(H)\right]^{2}+\frac{l^{\prime}}{l}\left[L_{l-1}^{l^{\prime}-l}(H)\right]^{2}$ as well as $F\left(l, l^{\prime}, H\right)=1$ for $l=0$, where the function $L_{n}^{\alpha}(x)$ stands for the generalized Laguerre polynomial. In the limit of $e B \gg T^{2}, e^{-K} \approx 1$, the result in Eq. (48) is consistent with that in Eq. (46). In this work, we take thermal averaged relaxation time $\left\langle\tau_{B, q}\right\rangle$ of quarks as dynamic input for the computation of thermoelectric coefficients, which is defined as

$$
\left\langle\tau_{B, q}\right\rangle\left(T, \mu_{q}\right)=\frac{1}{\left\langle\tau_{B, q}^{-1}\right\rangle}=\frac{\sum_{l=0}^{\infty} \int_{-\infty}^{+\infty} d p_{z} \bar{f}_{B, q(\bar{q})}^{0}\left(p_{z}\right)}{\sum_{l=0}^{\infty} \int_{-\infty}^{+\infty} d p_{z} \tau_{B, q}^{-1}\left(p_{z}\right) \bar{f}_{B, q}^{0}\left(p_{z}\right)} .
$$

In the anisotropic medium, the $\xi^{\prime}$-dependent thermal averaged relaxation time of quark, $\left\langle\tau_{B, q}^{\xi^{\prime}}\right\rangle$, also can be obtained by substituting $\bar{f}_{B, q}^{0}$ and $\tau_{B, q}^{-1}$ in Eq. (50) with $\bar{f}_{B, q}^{\xi^{\prime}}$ and $\left(\tau_{B, q}^{\xi^{\prime}}\right)^{-1}$, respectively. And in the numerical calculation we artificially truncate the sum of Landau levels at a finite maximum $l$.

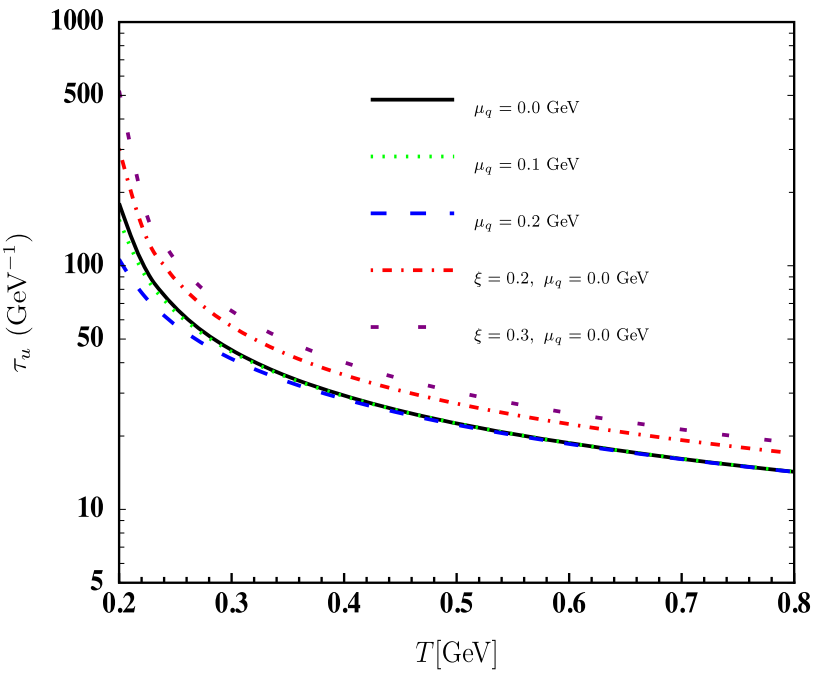

Fig. 1 The temperature dependence of the thermal relaxation time for $u$ quarks $\left(\tau_{u}\right)$ at different quark chemical potentials, viz, $\mu_{q}=0.0 \mathrm{GeV}$ (black solid line), $0.1 \mathrm{GeV}$ (green dotted line) and $0.2 \mathrm{GeV}$ (blue dashed line) for $B=0$. The estimation of $\tau_{u}$ at $\mu_{q}=0.0 \mathrm{GeV}$ is also extended to the anisotropic medium induced by initial spatial expansion with $\xi=0.2$ (red dotted-dashed line) and 0.3 (purple short-dashed line)

\section{Numerical result and discussion}

In the numerical calculation, we use the current masses of three-flavor quarks $\left(m_{u}=3 \mathrm{MeV}, m_{d}=5 \mathrm{MeV}\right.$ and $\left.m_{s}=80 \mathrm{MeV}\right)$ as input parameters. As mentioned in Sect. 1, under the weak magnetic field $\left(g^{2} T^{2}>e B\right)$ all scatterings of partons in the QGP are unaffected by magnetic field, thus the calculation of the relaxation time remains the same as in the absence of the magnetic field. In Fig. 1, the thermal behavior of the relaxation time for $u$-quarks $\left(\tau_{u}\right)$ at various quark chemical potentials $\left(\mu_{q}\right)$ and anisotropic parameters $(\xi)$ is displayed. We observe that $\tau_{u}$ decreases with increasing temperature and the order of magnitude of $\tau_{u}$ at small temperature is much larger than that at high temperature. We also notice $\tau_{u}$ decreases as $\mu_{q}$ increases at small temperature, whereas the decreasing feature of $\tau_{u}$ with $\mu_{q}$ is marginal at high temperature $(T>0.4 \mathrm{GeV})$. This is because that with increasing temperature the ratio $\mu_{q} / T$ becomes smaller, which leads to the result of different factors associated with $e^{ \pm \mu_{q} / T}$ in Eq. (40) is nearly $\mu_{q}$-independent. From Fig. 1 we also can clearly see that $\tau_{u}$ in an anisotropic QGP $(\xi=0.1)$ has an overall improvement compared to that in an isotropic QGP, and the degree of improvement can be further strengthened with the increase of $\xi$. To better understand the qualitative and quantitative behaviors of the total Seebeck coefficient $\left(S_{x x}\right)$ and the total Nernst signal $(N)$ for weak magnetic field, we first present our results of the total electrical $\left(\sigma_{x x}, \sigma_{x y}\right)$ and thermoelectric $\left(\alpha_{x x}, \alpha_{x y}\right)$ conductivity tensors. In Fig. 2a, we perform the temperature dependence of the total electrical conductivity $\left(\sigma_{x x}\right)$ at $\mu_{q}=0.1 \mathrm{GeV}$ for 

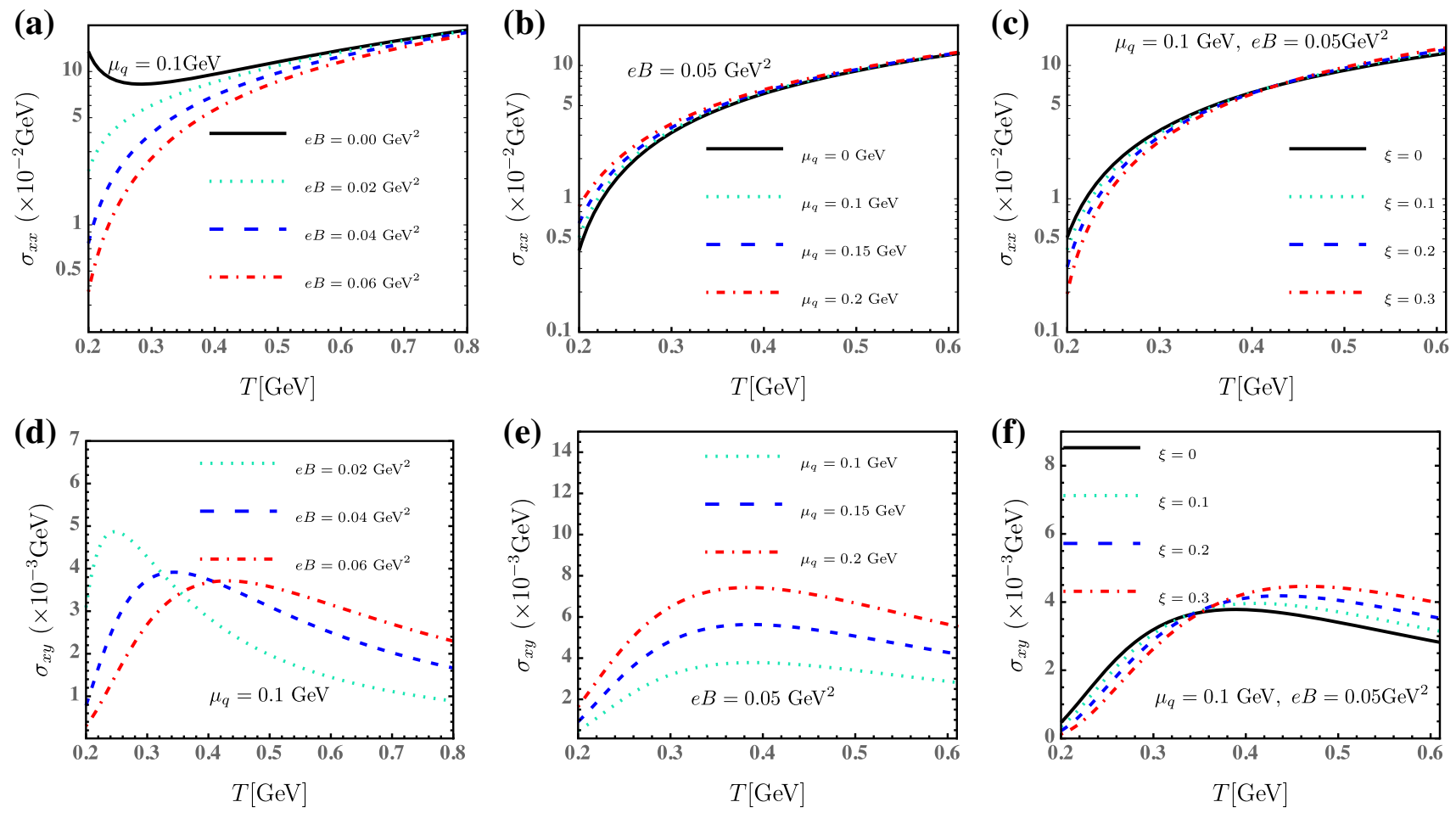

Fig. 2 a, d The temperature dependences of the electrical conductivity $\left(\sigma_{x x}\right)$ and the Hall conductivity $\left(\sigma_{x y}\right)$ for $\mu_{q}=0.1 \mathrm{GeV}$ at different weak magnetic fields, namely, $e B=0 \mathrm{GeV}^{2}$ (black solid line), $0.02 \mathrm{GeV}^{2}$ (cyan dotted lines), $0.04 \mathrm{GeV}^{2}$ (blue dashed lines), $0.06 \mathrm{GeV}^{2}$ (red dotted-dashed lines). b, e The temperature dependences of $\sigma_{x x}$ and $\sigma_{x y}$ for $e B=0.05 \mathrm{GeV}^{2}$ at $\mu_{q}=0 \mathrm{GeV}$ (black solid line),
$0.1 \mathrm{GeV}$ (cyan dotted lines), $0.15 \mathrm{GeV}$ (blue dashed lines) and $0.2 \mathrm{GeV}$ (red dotted-dashed lines). c, f The temperature dependences of $\sigma_{x x}$ and $\sigma_{x y}$ for $\mu_{q}=0.1 \mathrm{GeV}$ and $e B=0.05 \mathrm{GeV}^{2}$ in a weakly anisotropic medium with $\xi=0$ (black solid lines), 0.1 (cyan dotted lines), 0.2 (blue dashed lines), 0.3 (red dotted-dashed lines) different weak magnetic fields. At the vanishing magnetic field, the thermal evolution of $\sigma_{x x, q_{f}}$ for $f$ th flavor is basically dominated by the multiplicative result of factors, i.e., the relaxation time $\left(\tau_{q_{f}}\right)$ and quark distribution function $\left(\bar{f}_{q_{f}}^{0}\right)$ in the integrand of Eq. (23). At small $T$, the sharply decreasing feature of $\tau_{q_{f}}(T)$ significantly wins over the increasing feature of $\bar{f}_{q_{f}}^{0}(T)$, therefore the total electrical conductivity $\left(\sigma_{x x}\right)$ decreases with increasing temperature. However, at high $T$, the increasing behavior of $\bar{f}_{q_{f}}^{0}(T)$ is more prominent than the decreasing behavior of $\tau_{q_{f}}(T)$. As a result, $\sigma_{x x}$ for vanishing magnetic field decreases at small $T$ then increases at high $T$, as shown in Fig. 2a. In the presence of $B$, we observe that $\sigma_{x x}$ has a suppression compared to that in the vanishing $B$. This is due to that a additional factor, $1 /\left(1+\left(\omega_{c, q_{f}} \tau_{q_{f}}\right)^{2}\right)$, in integrand of Eq. (23) is always less than 1 . We see that $\sigma_{x x}$ monotonously increases with increasing $T$ at nonzero $B$, which is different with the thermal behavior of $\sigma_{x x}$ at zero field. This can be understood as follows: The qualitative behavior of $\sigma_{x x, q_{f}}$ for $f$ th flavor at weak $B$ is basically coming from the interplay between $\tau_{q_{f}} /\left(1+\left(\omega_{c, q_{f}} \tau_{q_{f}}\right)^{2}\right)$ and $\bar{f}_{q_{f}}^{0}$. At small $T, \tau_{q_{f}}$ is large and $\sigma_{x x}(T) \sim \sum_{f} \tau_{q_{f}} \bar{f}_{q_{f}}^{0} /\left(1+\left(\omega_{c, q_{f}} \tau_{q_{f}}\right)^{2}\right) \sim \sum_{f} \bar{f}_{q_{f}}^{0} / \tau_{q_{f}}$, consequently, $\sigma_{x x}$ increases with increasing $T$. At high $T$, $\tau_{q_{f}}$ is relatively small, $\tau_{q_{f}} /\left(1+\left(\omega_{c, q_{f}} \tau_{q_{f}}\right)^{2}\right) \sim \tau_{q_{f}}$, the thermal behavior of $\sigma_{x x}$ at weak field is consistent with the counterpart at zero field. Alternatively, the dependence of $\sigma_{x x}$ on $B$ only arises from the cyclotron frequency $\left(\omega_{c, q_{f}}\right)$. Therefore, $\sigma_{x x}$ decreases as $B$ grows at small $T$ due to $\sigma_{x x}(B) \sim \sum_{f} \frac{1}{\omega_{c, q_{f}}^{2}}$, and the positive effect of $B$ on $\sigma_{x x}$ is unconspicuous at high $T$ due to $\sigma_{x x}(B) \sim$ constant. Next, we consider the effect of quark chemical potential $\left(\mu_{q}\right)$ on the estimations of the conductivity tensors at $e B=0.05 \mathrm{GeV}^{2}$. We remind the reader that at finite $\mu_{q}$ due to the number density of quarks is always larger than that of anti-quarks in the QGP, the contribution of quarks to the tensors in magnitude is prominent. At relatively small temperature, $\sigma_{x x}\left(\mu_{q}\right) \sim \sum_{f} \bar{f}_{q_{f}}^{0}\left(\mu_{q}\right) / \tau_{q_{f}}\left(\mu_{q}\right)$, where both $\bar{f}_{q_{f}}^{0}\left(\mu_{q}\right)$ and $1 / \tau_{q_{f}}\left(\mu_{q}\right)$ are increasing functions. Whereas, at high $T, \tau_{q_{f}}$ is nearly unchanged with the variation of $\mu_{q}$ as shown in Fig. 1, thus $\sigma_{x x}\left(\mu_{q}\right) \sim \sum_{f} \bar{f}_{q_{f}}^{0}\left(\mu_{q}\right)$. As the ratio $\mu_{q} / T$ at high $T$ is small, the Boltzmann factor $e^{\mu_{q} / T}$ in quark distribution increases insignificantly with an increase of $\mu_{q}$. As a result, with the increase of $\mu_{q}, \sigma_{x x}$ increases at $T<0.3 \mathrm{GeV}$ then remains almost constant at $T>0.3 \mathrm{GeV}$, as shown in 
Fig. 2b. The momentum anisotropy induced by initial spatial expansion is also considered in the estimation of the tensors. In Fig. 2c, the $T$ dependence of $\sigma_{x x}$ at $\mu_{q}=0.1 \mathrm{GeV}$ and $e B=0.05 \mathrm{GeV}^{2}$ in the weakly anisotropic QGP (we take $\xi=0.1,0.2$ and 0.3 ) is performed. As illustrated in Fig 2c, the thermal behavior of $\sigma_{x x}$ in an anisotropic medium is consistent with the counterpart in an isotropic medium. However, the dependence of $\sigma_{x x}$ on $\xi$ is nonmonotonic in the entire $T$ domain of interest, which can be easily understood from the expression of $\sigma_{x x, q_{f}}$ in Eq. (22). Since the first term in Eq. (22) is numerically much larger than the second term, thus the $\xi$ dependence of $\sigma_{x x, q_{f}}$ is mainly determined by $\frac{\tau_{q_{f}}(\xi)}{1+\omega_{c, q_{f}}^{2} \tau_{q_{f}}(\xi)^{2}}(1+\xi)$ in the first term. At small $T, \sigma_{x x}(\xi) \sim \sum_{f}(1+\xi) / \tau_{q_{f}}(\xi)$, where the increasing feature of $(1+\xi)$ is compensated by the decreasing feature of $1 / \tau_{q_{f}}(\xi)$, leading $\sigma_{x x}$ as a decreasing function of $\xi$. At high $T, \sigma_{x x}(\xi) \sim \sum_{f} \tau_{q_{f}}(\xi)(1+\xi), \sigma_{x x}$ increases as $\xi$ grows.

Due to the absence of the Hall effect at the vanishing magnetic field, the calculation of the total Hall conductivity $\left(\sigma_{x y}\right)$ is only performed in the magnetic background field. In the isotropic QGP, for the Hall conductivity of $f$ th flavor quarks, $\sigma_{x y, q_{f}}$, its thermal behavior is mainly dominated by the form factor $\omega_{c, q_{f}} \tau_{q_{f}}^{2} /\left(1+\left(\omega_{c, q_{f}} \tau_{q_{f}}\right)^{2}\right)$ and associated quark distribution $\left(\bar{f}_{q_{f}}^{0}\right)$ in Eq. (23). The numerator of the form factor reminds us that the sign of $\sigma_{x y, q_{f}}$ is dependent of quark type. Due to the discrepancies of various flavor quarks in fractional charge value $\left(q_{u, d, s}=2 / 3,-1 / 3,-1 / 3\right)$ and mass, $\sigma_{x y, u}$ is greater than $\sigma_{x y, d}+\sigma_{x y, s}$. This is why the sign of $\sigma_{x y}$ always remains positive. In Fig. $2 \mathrm{~d}$ we see that $\sigma_{x y}$ at a fixed $B$ for $\mu_{q}=0.1 \mathrm{GeV}$ exhibits a nonmonotonic thermal behavior. More exact, $\sigma_{x y}(T)$ first increases, reaches a maximum then decreases. This peak structure of $\sigma_{x y}(T)$ has also been observed in Ref. [34]. We can understand this behavior in the following way. At small $T, \sigma_{x y}(T) \sim \sum_{f} \bar{f}_{q_{f}}^{0}$, where $\bar{f}_{q_{f}}^{0}$ is an increasing function of $T$. At high $T, \sigma_{x y}(T) \sim \sum_{f} \tau_{q_{f}}^{2} \bar{f}_{q_{f}}^{0}$ and the decreasing feature of $\tau_{q_{f}}^{2}(T)$ greatly overwhelms the increasing feature of $\bar{f}_{q_{f}}^{0}(T)$, leading $\sigma_{x y}$ as a decreasing function of $T$. From Fig. $2 \mathrm{~d}$ we also see that as $B$ rises, $\sigma_{x y}$ decreases at small $T$ due to $\sigma_{x y}(B) \sim 1 / B$, and increases at high $T$ due to $\sigma_{x y}(B) \sim B$. Moreover, the position for the maximum of $\sigma_{x y}$ shifts toward higher $T$ with the increase in $B$. In Fig. 2e, $\sigma_{x y}$ increases as $\mu_{q}$ grows, which can be well understood from the behavior of prominent $\sigma_{x y, u}\left(\mu_{q}\right)$. In the entire $T$ domain of interest, the qualitative behavior of $\sigma_{x y, u}\left(\mu_{q}\right)$ is almost determined by quark distribution function. Compared to $\sigma_{x x}\left(\mu_{q}\right)$, we note that the effect of $\mu_{q}$ on $\sigma_{x y}$ is obvious at high temperature, which is attributed to the increment in quark distribution function with the increase of $\mu_{q}$ is comparable to the value of $\sigma_{x y}$ itself. In the momentum anisotropic medium, the absolute value of $\sigma_{x y, q_{f}}$ for various flavors increases monotonously with the increase of $\xi$ because $\frac{\left|\omega_{c, q_{f}}\right| \tau_{q_{f}}(\xi)^{2}(1+\xi)}{1+\left(\omega_{c, q_{f}} \tau_{q_{f}}(\xi)\right)^{2}}$ in Eq. (22) is an increasing function of $\xi$. Although $\sigma_{x y, u}$ itself is relatively larger than $\sigma_{x y, d}+\sigma_{x y, s}$ in magnitude, the variation in $\sigma_{x y, d}+\sigma_{x y, s}$ with $\xi$ is numerically stronger (weaker) than the variation in $\sigma_{x y, u}$ with $\xi$ at $T<0.3 \mathrm{GeV}$ (at $T>0.3 \mathrm{GeV}$ ) (we don't display the figure, but it's a truth). Hence, with the increase of $\xi, \sigma_{x y}$ first decreases at relatively low $T$ then increases at high $T$, as shown in Fig. 2f. We also observe that as $\xi$ grows, the maximum of $\sigma_{x y}$ increases and shifts towards higher temperature.

For the total thermoelectric conductivity $\left(\alpha_{x x}\right)$, the computation is also limited to nonzero chemical potential case. From a quantitative respect, the first term in Eq. (25) is numerically larger than the second term. This mathematical difference arises from the different power of momentum in respective integrands. From a qualitative respect, for the thermoelectric conductivity of $f$ th flavor quarks, $\alpha_{x x, q_{f}}$, its thermal behavior under nonzero $B$ and nonzero $\mu_{q}$ is determined by $\frac{q_{f} \tau_{q_{f}}}{1+\left(q_{f} \omega_{c, q_{f}} \tau_{q_{f}}\right)^{2}}$ and $\bar{f}_{q_{f}}^{0}$ in Eq. (25), as well as its sign depends on the quark type. Thus the absolute $\alpha_{x x, q_{f}}$ for various flavors is similar to $\sigma_{x x, q_{f}}$ in the qualitative and quantitative. However, because of the sensitivity of $\alpha_{x x, q_{f}}$ in charge characteristic and mass, the qualitative and quantitative behavior of the total thermoelectric conductivity $\left(\alpha_{x x}\right)$ is different to that of the total electrical conductivity $\left(\sigma_{x x}\right)$. In Fig $3 \mathrm{a}, \alpha_{x x}$ at a nonzero $B$ exhibits negative in sign. ${ }^{1}$ At high enough temperature, $\mu_{q} / T \sim 0$, the Boltzmann factor $e^{\mu_{q} / T}$ in $\bar{f}_{q_{f}}^{0}$ becomes smaller, the numerical difference between $\alpha_{x x, d}+\alpha_{x x, s}$ and $\alpha_{x x, u}$ reduces gradually. As a result, $\alpha_{x x}$ is closer to zero with increasing $T$, as illustrated in Fig. 3a. And with the increase of $B$, we clearly see that the thermal behavior of $\alpha_{x x, q_{f}}$ exhibit a valley structure. The $\mu_{q}$ and $\xi$ dependence on the absolute $\alpha_{x x}$ in the entire $T$ domain of interest is similar to the counterpart on $\sigma_{x x}$. Furthermore, as $\xi$ grows, the minimum of $\alpha_{x x}$ shifts towards higher temperature.

For the total Hall-like thermoelectric conductivity $\left(\alpha_{x y}\right)$, the estimation is limited to the case of nonzero $B$, which can be easily understood from the corresponding expression in Eq. (25). Similar to $\alpha_{x x}$, the numerical value of $\alpha_{x y}$ for a fixed $\mu_{q}$ is also mainly determined by the first term in Eq. (25), the difference is that the sign of $\alpha_{x y}$ is independent of quark type and always remains positive. Since the thermal behavior of $\alpha_{x y, q_{f}}$ mainly depends on $\frac{q_{f} \omega_{c, q_{f}} \tau_{q_{f}}^{2}}{1+\left(\omega_{c, q_{f}} \tau_{q_{f}}\right)^{2}}$ and $\bar{f}_{q_{f}}^{0}$ in Eq. (25), the temperature and magnetic field dependences of $\alpha_{x y}$ are allied to that of $\sigma_{x y}$, as shown in Fig. 3d. In Fig. 3e, we clearly observe that $\alpha_{x y}$ decreases as $\mu_{q}$ increases at small $T$, which

\footnotetext{
1 In this paper, the sign of $\alpha_{x x}$ for a fixed $\mu_{q}$ at the vanishing magnetic field exhibits positive. However, we do not discuss much $\alpha_{x x}$ in the vanishing magnetic field qualitatively or quantitatively due to it's marginal results $\left(10^{-4} \sim 10^{-5}\right)$ at $0.2 \mathrm{GeV}<T<0.4 \mathrm{GeV}$.
} 

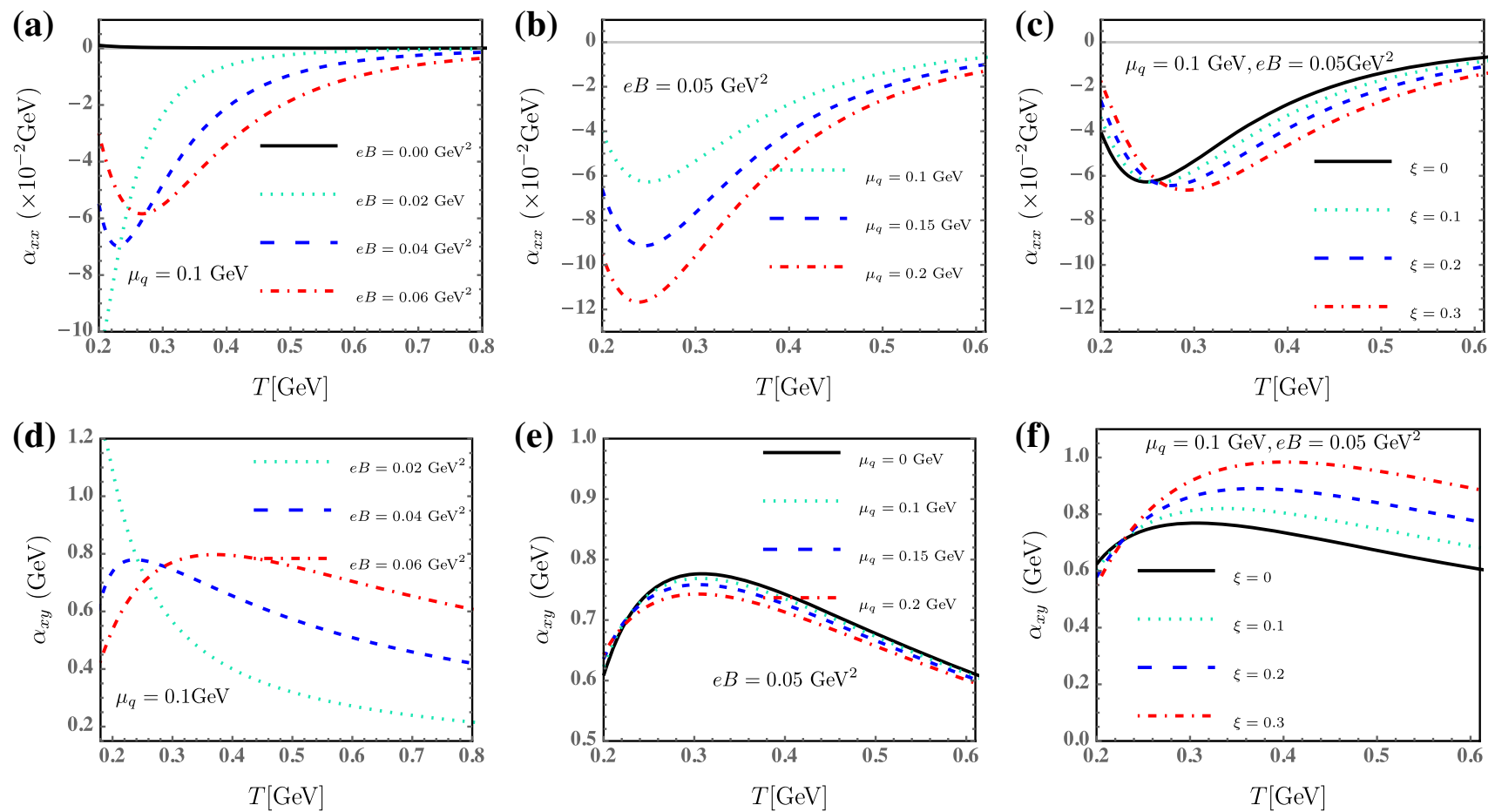

Fig. 3 a, $\mathbf{d}$ The temperature dependences of the thermoelectric conductivity $\left(\alpha_{x x}\right)$ and the Hall-like thermoelectric conductivity $\left(\alpha_{x y}\right)$ for $\mu_{q}=0.1 \mathrm{GeV}$ at different magnetic fields, namely, $e B=0 \mathrm{GeV}^{2}$ (black solid line), $0.02 \mathrm{GeV}^{2}$ (cyan dotted lines), $0.04 \mathrm{GeV}^{2}$ (blue dashed lines), $0.06 \mathrm{GeV}^{2}$ (red dotted-dashed lines). b, e The temperature dependences of $\alpha_{x x}$ and $\alpha_{x y}$ for $e B=0.05 \mathrm{GeV}^{2}$ at $\mu_{q}=0 \mathrm{GeV}$ (black solid line), $0.1 \mathrm{GeV}$ (cyan dotted lines), $0.15 \mathrm{GeV}$ (blue dashed lines) and $0.2 \mathrm{GeV}$ (red dotted-dashed lines). c, f The temperature dependences of $\alpha_{x x}$ and $\alpha_{x y}$ for $\mu_{q}=0.1 \mathrm{GeV}$ and $e B=0.05 \mathrm{GeV}^{2}$ in a weakly anisotropic QGP induced by initial spatial expansion with $\xi=0$ (black solid lines), 0.1 (cyan dotted lines), 0.2 (blue dashed lines), 0.3 (red dotted-dashed lines) is opposite to the behavior of $\sigma_{x y}\left(\mu_{q}\right)$. This is attributed that at small $T$ though the first term in the expression of $\alpha_{x y}$ is much larger than the second term in magnitude, the increment of the first term with the increase of $\mu_{q}$ is compensated by the more significant reduction of the second term. At high $T, \alpha_{x y}$ remains almost constant with the variation of $\mu_{q}$ because $\mu_{q} / T$ at high $T$ is small, the variation of $e^{ \pm \mu_{q} / T}$ in distribution functions is negligible compared to the value of $\alpha_{x y}$ itself. In the anisotropic medium, $\frac{q_{f} \omega_{c, q_{f}} \tau_{q_{f}}(\xi)^{2}}{1+\omega_{c, q_{f}}^{2} \tau(\xi)^{2}}$ for $f$ th quarks in Eq. (24) is an increasing function of $\xi$ at any given $T$. In addition, the variation of the first term for $\alpha_{x y}$ in Eq. (24) with $\xi$ is more greater than the counterpart of the second term. Therefore, $\alpha_{x y}$ increases as $\xi$ increases in the entire $T$ domain of interest, as shown in Fig. $3 \mathrm{f}$.

Since the quantitative and qualitative behaviors of both the total Seebeck coefficient $\left(S_{x x}\right)$ and the total Nernst signal $(N)$ are attributed by the intricate interplay of four conductivity tensors, we only phenomenologically discuss the impacts of magnetic field $(B)$, quark chemical potential $\left(\mu_{q}\right)$, and anisotropic parameter $(\xi)$ on $S_{x x}$ and $N$ step by step. In Fig. 4a, we display the temperature dependence of $S_{x x}$ for finite $B$ at $\mu_{q}=0.1 \mathrm{GeV}$. In the semiconductor, a positive (negative) $S_{x x}$ implies that the generated electric current runs toward (away from) the direction of high temperature end in an electron (hole) rich side. In other word, $S_{x x}$ is negative for negatively charge carriers and positive for positively charge carriers. Similarly, in the QGP, the sign of $S_{x x}$ is positive (negative), indicating that the major carriers who dominate the conversion from a temperature gradient to an electric field are positively (negatively) charged quarks. In our work, the sign of $S_{x x}$ in the QGP for $\mu_{q}=0.1 \mathrm{GeV}$ at zero $B$ is positive and the numerical values of $S_{x x}$ are in $0.0006<S_{x x}<0.003$ at $0.2 \mathrm{GeV} \leqslant T \leqslant 0.4 \mathrm{GeV}$. It's worth noting that our results are close to the results in Ref. [61], where the values of $S_{x x}$ for the QGP at the vanishing magnetic field for $\mu_{B}=0.05 \mathrm{GeV}$ lie in the regime of $0<S_{x x}<0.005$ under the same temperature region. At the nonzero (zero) magnetic field, the sign of $S_{x x}$ in the QGP is negative (positive), which is consistent with the sign of $\alpha_{x x}$. But the thermal behavior of $S_{x x}$ is monotonic instead of nonmonotonic. At high enough $T, S_{x x}$ approaches to zero, indicating the system along $x$-axis is close to an isothermal state. And the absolute value of $S_{x x}$ in the QGP increase as $B$ increases. From Fig. $4 b$, c, we observe that the absolute $S_{x x}$ significantly increases as $\mu_{q}$ and $\xi$ increase at $T<0.4 \mathrm{GeV}$. Next, we start to discuss the Nernst effect. 

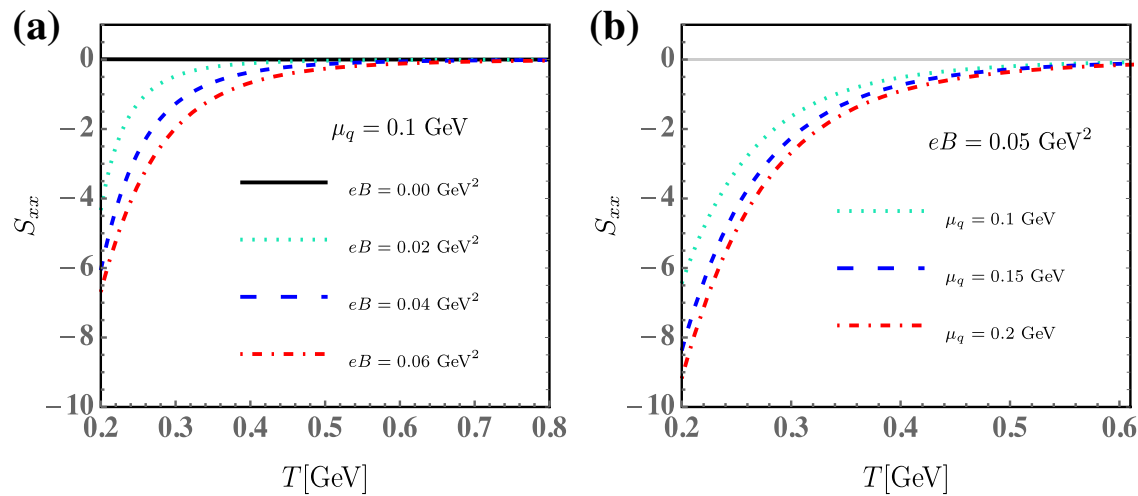

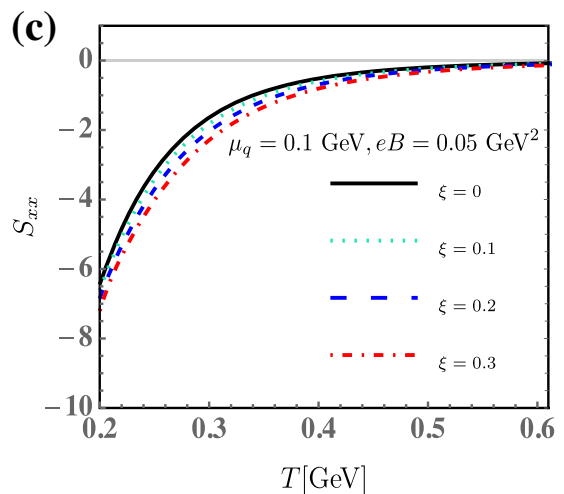

Fig. 4 a The temperature dependence of the Seebeck coefficient $\left(S_{x x}\right)$ for $\mu_{q}=0.1 \mathrm{GeV}$ at different magnetic fields, namely $e B=0 \mathrm{GeV}^{2}$ (black solid line), $0.02 \mathrm{GeV}^{2}$ (cyan dotted line), $0.04 \mathrm{GeV}^{2}$ (blue dashed line), $0.06 \mathrm{GeV}^{2}$ (red dotted-dashed line). b The temperature dependence of $S_{x x}$ for $e B=0.05 \mathrm{GeV}^{2}$ at $\mu_{q}=0.1 \mathrm{GeV}$ (cyan dotted line),
$0.15 \mathrm{GeV}$ (blue dashed line) and $0.2 \mathrm{GeV}$ (red dotted-dashed line). $\mathbf{c}$ The temperature dependence of $S_{x x}$ for $\mu_{q}=0.1 \mathrm{GeV}$ and $e B=0.05 \mathrm{GeV}^{2}$ in a weakly anisotropic medium with $\xi=0$ (black solid line), 0.1 (cyan dotted line), 0.2 (blue dashed line), 0.3 (red dotted-dashed line) (a)

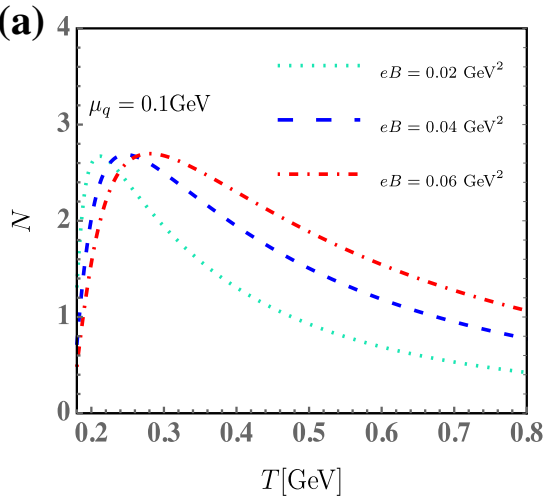

(b)

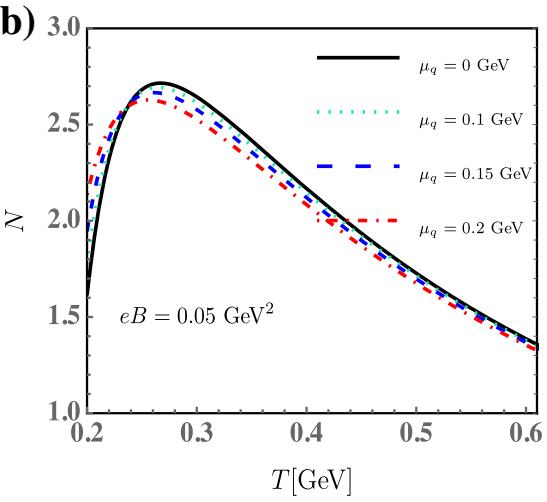

(c)

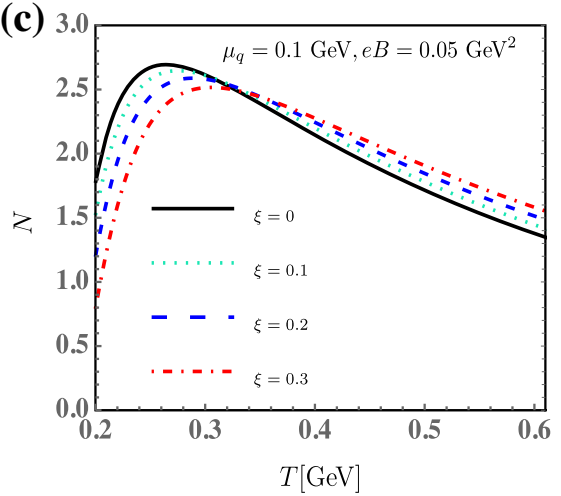

Fig. 5 a The temperature dependence of the Nernst signal $(N)$ for $\mu_{q}=0.1 \mathrm{GeV}$ at different magnetic fields, namely $e B=0.02 \mathrm{GeV}^{2}$ (cyan dotted line), $0.04 \mathrm{GeV}^{2}$ (blue dashed line), $0.06 \mathrm{GeV}^{2}$ (red dotteddashed line). $\mathbf{b}$ The temperature dependence of $N$ for $e B=0.05 \mathrm{GeV}^{2}$ at $\mu_{q}=0 \mathrm{GeV}$ (black solid line), $0.1 \mathrm{GeV}$ (cyan dotted line), $0.15 \mathrm{GeV}$ (blue dashed line) and $0.2 \mathrm{GeV}$ (red dotted-dashed line). c The temperature dependence of $N$ for $\mu_{q}=0.1 \mathrm{GeV}$ and $e B=0.05 \mathrm{GeV}^{2}$ in a weakly anisotropic medium with $\xi=0$ (black solid line), 0.1 (cyan dotted line), 0.2 (blue dashed line), 0.3 (red dotted-dashed line)
For vanishing $B$, there is no Lorentz force to bend the trajectories of the thermally diffusing charge carriers, so the Nernst effect is absent. Figure 5a shows the evolution of $N$ as a function of $T$ at different $B$ for $\mu_{q}=0.1 \mathrm{GeV}$. Unlike $S_{x x}, N$ in sign is independent of the charge carrier type, which can well understand from the associated expression. We can clearly see the value of $N$ always remains positive and the thermal evolution of $N$ at the magnetic field has a peak structure. We also observe as $B$ grows, $N$ decreases at small $T$ whereas increases at high $T$, which is consistent with $\sigma_{x y}(B)$ and $\alpha_{x y}(B)$ in the qualitative. In this work, the maximum of $N$ for $\mu_{q}=0.1 \mathrm{GeV}$ approximately is 2.8 . We can zoom in Fig. $5 \mathrm{~b}$ and observe that the $\mu_{q}$ dependence of $N$ is very similar to $\alpha_{x y}\left(\mu_{q}\right)$, except that $N$ weakly decreases as $\mu_{q}$ increases at $T<0.26 \mathrm{GeV}$. Anyway, the effect of $\mu_{q}$ on $N$ is not obvious. The effect of momentum anisotropy induced by initial spatial expansion on $N$ at $\mu_{q}=0.1 \mathrm{GeV}$ for $e B=0.05 \mathrm{GeV}^{2}$ is plotted in Fig. 5c. We see that as $\xi$ increases, $N$ decreases at relatively low temperature then increases at high temperature, which is qualitatively akin to $\sigma_{x y}(\xi)$. Furthermore, with the rise in $\xi$, the maximum of $N$ falls and slightly shifts to higher temperature.

The investigation of the thermoelectric coefficient is also converted to the strong magnetic background field. The calculation of the longitudinal conductivity tensors is first performed under the LLL approximation. In the LLL approximation, $2 \rightarrow 1$ scattering process and $2 \rightarrow 2$ scattering process are taken into account. Although the inverse relaxation times in Eqs. (46) and (47) for two kind of processes are of the same order of $\alpha_{s, B}$, the numerical value of inverse relaxation time for $2 \rightarrow 2$ process is marginal compared with that for $2 \rightarrow 1$ process. Since the largest 


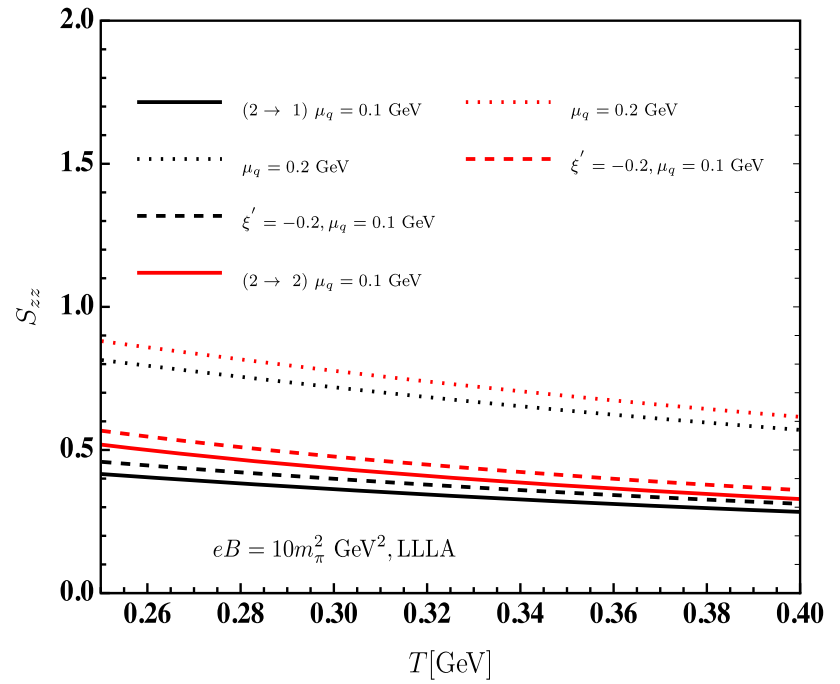

Fig. 6 The temperature dependence of Seebeck coefficient along the direction of magnetic field $\left(S_{z z}\right)$ for $2 \rightarrow 1$ process (black lines) and $2 \rightarrow 2$ process (red lines) in the LLL approximation. Solid lines and dotted lines represent the calculations of $S_{x x}$ for $e B=10 m_{\pi}^{2} \mathrm{GeV}^{2}$ are performed at $\mu_{q}=0.1 \mathrm{GeV}$ and at $0.2 \mathrm{GeV}$, respectively. Dashed lines denote the temperature dependence of $S_{x x}$ at $\mu_{q}=0.2 \mathrm{GeV}$ for $e B=$ $10 m_{\pi}^{2} \mathrm{GeV}^{2}$ in an anisotropic medium induced by strong magnetic field (we take $\xi^{\prime}=-0.2$ ). Within the assumed regime $m_{q}^{2} \ll \alpha_{s, B} e B \lesssim$ $T^{2} \lesssim e B$, we artificially take $e B=10 m_{\pi}^{2} \mathrm{GeV}^{2}$ and the appropriate temperature can be concentrated within the region $0.25 \mathrm{GeV} \leqslant T \leqslant$ $0.4 \mathrm{GeV}$

inverse relaxation time determines the final inverse relaxation time, the $2 \rightarrow 1$ process is significantly dominated over $2 \rightarrow 2$ process in the strong magnetized QGP. Nevertheless, we still can compute the respective contribution to the component of the longitudinal Seebeck coefficient, namely, $S_{z z}$, in the LLL approximation. Figure 6 demonstrates that $S_{z z}$ of isotropic QGP in the LLL approximation for $\mu_{q}=0.1 \mathrm{GeV}$ decreases with increasing temperature. Unlike $S_{x x}$ at weak magnetic field, the sign of $S_{z z}$ at strong magnetic field within the LLL approximation is positive, indicating that the dominant charge carriers for converting the thermal gradient along the direction of magnetic field to electric field are positively charged quarks. Yet, in Ref. [64] the result of $S_{z z}$ is negative because the sensitivity of the relaxation time in the quark chemical potential is not taken into account. We also observe the value of $S_{z z}$ for $2 \rightarrow 1$ process is comparable with that for $2 \rightarrow 2$ process. Actually, $S_{z z}$ in the LLL approximation is independent of $B$ due to the fact that the $B$-dependent factors in the numerator of $S_{z z}$ and the counterparts in the denominator cancel out. Similar to the previous calculation in weak magnetic field, we also consider the effect of quark chemical potential $\left(\mu_{q}\right)$ on $S_{z z}$ in the LLL approximation. As illustrated in Fig. 6, $S_{z z}$ for both $2 \rightarrow 1$ process and $2 \rightarrow 2$ process numerically increases as $\mu_{q}$ increases in entire $T$ domain of interest. In comparison to the isotropic medium, $S_{z z}$ in a strong magnetic field- driven anisotropic medium (we take $\xi^{\prime}=-0.2$ ) remains unchanged in the qualitative but has a quantitative enhancement. Finally, the calculation of $S_{z z}$ is also extended to a more realistic regime $g^{2} T^{2} \ll e B$ in which the contribution from higher Landau levels (hLLs) are considered. In Fig. 7a and $\mathrm{b}$, as Landau level $(l)$ increases, we note that the scaled longitudinal electrical conductivity $\left(\sigma_{z z} / T\right)$ increases, whereas the scaled longitudinal thermoelectric conductivity $\left(\alpha_{z z} / T\right)$ decreases. In our work, the numerical values of $\sigma_{z z} / T$ at $e B=10 m_{\pi}^{2} \mathrm{GeV}^{2}$ and $\mu_{q}=0 \mathrm{GeV}$ within the consideration of hLLs contribution are in $0.3<\sigma_{z z} / T<0.7$ for $0.2 \mathrm{GeV} \leqslant T \leqslant 0.5 \mathrm{GeV}$, which is consistent with the result $\left(0.2<\sigma_{z z} / T<0.7\right)$ of existing report [42] in the same configuration. And in present work, $\sigma_{z z} / T$ within the effect of hLLs also quantitatively lies in the range of Lattice QCD results $\left(0.1 \leqslant \sigma_{e l} / T \leqslant 1.0\right)$ from Refs. [112-114]. In Fig. 7c, we see that the thermal behavior of $S_{z z}$ beyond the LLL approximation with $l=20$ is similar to the counterpart of $S_{z z}$ in the LLL approximation. Due to the decreasing features of both $\alpha_{z z}$ and $1 / \sigma_{z z}$ with increasing Landau level as shown in Fig. 7a, b, the value of $S_{z z}$ can be suppressed as the Landau level rises. And $S_{z z}$ even changes sign from positive to negative as the Landau level grows, which mimics the dependence of $\alpha_{z z}$ on the Landau level. The change of $S_{z z}$ in sign reflects the dominant charge carriers for converting the heat gradient along $z$-axis to electric field become negatively charged quarks rather than positively charged quarks as the increase of Landau level. Furthermore, at higher temperature, $S_{z z}$ for various Landau levels converges to zero, indicating that the system along $z$-axis is in a nearly isothermal state.

\section{Conclusion}

A theoretical investigation on the Seebeck effect and the Nernst effect of QGP in the magnetic fields has been presented. The associated Seebeck and Nernst signal are the functions regarding the electrical conductivity tensors and the thermoelectric conductivity tensors, which can be obtained by solving the relativistic Boltzmann equation under the relaxation time approximation. We found in the presence (absence) of weak magnetic field along $z$-axis, $S_{x x}$ for a nonzero quark chemical potential is negative (positive) in sign, indicating that the dominant charge carriers for converting heat gradient into electric field are negatively (positively) charged quarks. We found as temperature increases, $S_{x x}$ first decreases (absolute value increases) then gradually tends to zero, which implies that at high enough temperature the system reaches an isothermal state. And the absolute value of $S_{x x}$ has a further enhancement with the increase in magnetic field and quark chemical potential. We also extended the exploration to an anisotropic QGP, where the partons exhibit a local anisotropy $(\xi \neq 0)$ in the momentum space 
(a) $\quad \mu_{q}=0.1 \mathrm{GeV}, e B=10 m_{\pi}^{2} \mathrm{GeV}^{2}$

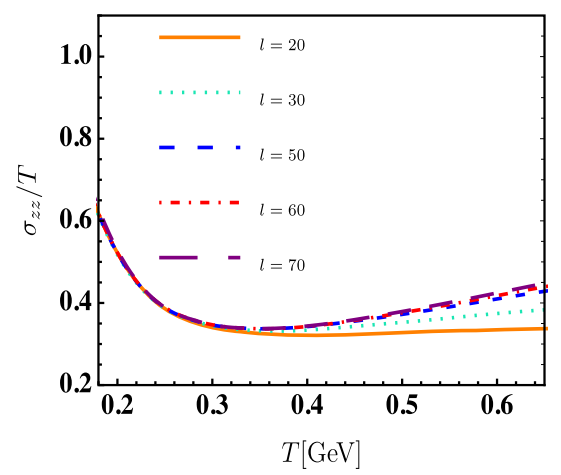

(b) $\quad \mu_{q}=0.1 \mathrm{GeV}, e B=10 m_{\pi}^{2} \mathrm{GeV}^{2}$

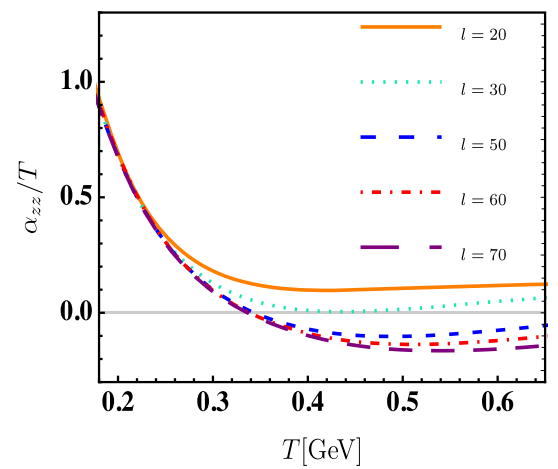

(c)

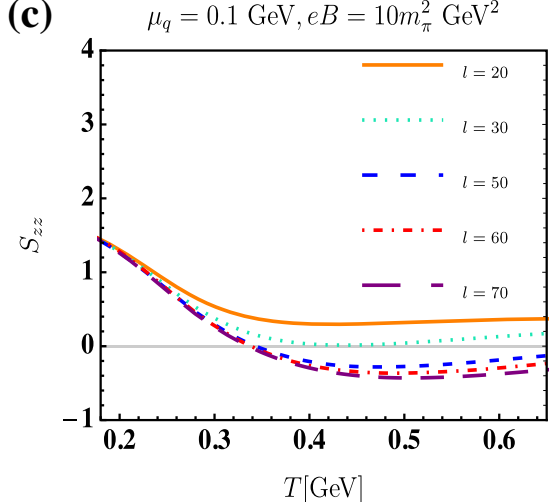

Fig. 7 The temperature dependences of the scaled longitudinal electrical conductivity $\left(\sigma_{z z} / T\right)(\mathbf{a})$, the scaled longitudinal thermoelectric conductivity $\left(\alpha_{z z} / T\right)(\mathbf{b})$ and the longitudinal Seebeck coefficient $\left(S_{z z}\right)$ (c) within different Landau levels ( $l=20$ (orange solid lines), 30 (cyan dotted lines), 50 (blue dashed lines), 60 (red dotted-dashed lines) and 70 (purple wide-dashed lines)) for $e B=10 m_{\pi}^{2} \mathrm{GeV}^{2}$ at $\mu_{q}=0.1 \mathrm{GeV}$ due to the rapid expansion of initial fireball along the beam direction. The results showed that the absolute value of $S_{x x}$ in a weakly anisotropic medium has an obvious enhancement compared to that in an isotropic medium $(\xi=0)$, and with the increase of $\xi$ this increment can be strengthened. Different from monotonous thermal behavior of $S_{x x}$, the temperature dependence of the Nernst signal $(N)$ for weak magnetic field exhibits a peak structure, and $N$ in sign is independent of the type of charge carriers. As magnetic field as well as anisotropic parameter $(\xi)$ increase, $N$ decreases at relatively small temperature whereas increases at high temperature. In contrast to the effects of magnetic field and momentum anisotropy, with the increase in quark chemical potential, $N$ increases at small temperature whereas decreases at high temperature.

In the strong magnetic field, the Seebeck coefficient along the direction of magnetic field, $S_{z z}$, has been calculated under the LLL approximation and beyond the LLL approximation. The value of $S_{z z}$ in the LLL approximation always remains positive and increases as quark chemical potential rises. And we found $S_{z z}$ in the LLL approximation is independent of the magnetic field strength. Under the same condition, the value of $S_{z z}$ for $2 \rightarrow 2$ process is comparable with that for $2 \rightarrow 1$ process, even though the former process is far less important than the latter process in the strongly magnetized QGP. In addition, $S_{z z}$ in the anisotropic QGP induced by strong magnetic field with $\xi^{\prime}=-0.2$ has an overall enhancement compared to that in the isotropic medium. With the increase of Landau level, $S_{z z}$ decreases and even changes the sign from positive to negative. For the future investigation, we may study the Seebeck coefficient and the Nernst signal at the magnetic field in the hadronic phase and near the transition phase region based on van der Waals hadron resonance gas (VDWHRG) model and QCD effective models (e.g., Polyakov Nambu-Jona-Lasinio model and Polyakov
Quark Meson model), respectively. Especially, a direct comparison of the results in the hadronic and partonic phases would be instructive.

Acknowledgements We thank Arpan Das for bringing some recent literature to our attention. This research is supported by the National Natural Science Foundation of China under Grant no. 11935007, Guangdong Major Project of Basic and Applied Basic Research No. 2020B0301030008, and the Fundamental Research Funds for the Central Universities under Grant no. 2020CXZZ107.

Data Availability Statement This manuscript has no associated data or the data will not be deposited. [Authors' comment: This is a theoretical work and no experimental data were used. The reader could contact the corresponding author for numerical results shown in this paper.]

Open Access This article is licensed under a Creative Commons Attribution 4.0 International License, which permits use, sharing, adaptation, distribution and reproduction in any medium or format, as long as you give appropriate credit to the original author(s) and the source, provide a link to the Creative Commons licence, and indicate if changes were made. The images or other third party material in this article are included in the article's Creative Commons licence, unless indicated otherwise in a credit line to the material. If material is not included in the article's Creative Commons licence and your intended use is not permitted by statutory regulation or exceeds the permitted use, you will need to obtain permission directly from the copyright holder. To view a copy of this licence, visit http://creativecomm ons.org/licenses/by/4.0/.

Funded by $\mathrm{SCOAP}^{3}$.

\section{Appendix A}

The collision term of species $a_{1}$ for the binary process $a_{1}\left(P_{1}\right)+a_{2}\left(P_{2}\right) \rightarrow a_{3}\left(P_{3}\right)+a_{4}\left(P_{4}\right)$ is given by $[115,116]$

$$
\begin{aligned}
C\left[f_{1}\right]= & \sum_{\text {pro }} \frac{d_{2}}{1+\delta_{34}} \frac{1}{2 \epsilon_{1}} \prod_{i=2}^{4} \int d \Gamma_{i}(2 \pi)^{4} \delta^{4}\left(P_{\text {tot }}\right)\left|\mathcal{M}^{\text {pro }}\right|^{2} \\
& \times\left[f_{1} f_{2}\left(1 \pm f_{3}\right)\left(1 \pm f_{4}\right)-f_{3} f_{4}\left(1 \pm f_{1}\right)\left(1 \pm f_{2}\right)\right] .
\end{aligned}
$$


In the above, $P_{\text {tot }}=P_{1}+P_{2}-P_{3}-P_{4} \cdot P_{i=1,2,3,4}=$ $\left(\epsilon_{i}, \mathbf{p}_{i}\right)$ denotes the four-momentum of particle, where $\epsilon_{i}=$ $\sqrt{\mathbf{p}_{i}^{2}+m_{i}^{2}}$. We use a notation $d \Gamma_{i}=\frac{d^{3} \mathbf{p}_{i}}{(2 \pi)^{3} 2 \epsilon_{i}}$ for convenience. The factor $1 /\left(1+\delta_{34}\right)$ is introduced to avoid double counting when particle $a_{1}$ and $a_{2}$ are identical. $\mathcal{M}^{\text {pro }}$ is the scattering amplitude for a specific binary process. Considering the distribution slightly derivate the equilibrium, hence the local momentum distribution of $i$ th species is given by $f_{i}=\bar{f}_{i}^{0}+\delta f_{i}=\bar{f}_{i}^{0}+\beta \bar{f}_{i}^{0}\left(1 \pm \bar{f}_{i}^{0}\right) \chi_{i}\left(\mathbf{p}_{i}\right)$, where $\chi_{i}$ is the response function in the effect of electric field. For (anti)quarks, the associated response functions hold the relation of $\chi_{\bar{q}}(\mathbf{p})=\chi_{q}(-\mathbf{p})$ due to the charge conjugation symmetry. However, for gluons $\chi_{g}$ is zero. Using the detailed balance condition $\bar{f}_{1}^{0} \bar{f}_{2}^{0}\left(1 \pm \bar{f}_{3}^{0}\right)\left(1 \pm \bar{f}_{4}^{0}\right)=\bar{f}_{3}^{0} \bar{f}_{4}^{0}\left(1 \pm \bar{f}_{1}^{0}\right)\left(1 \pm \bar{f}_{2}^{0}\right)$, the collision term can be rewritten as

$$
\begin{aligned}
C\left[f_{1}\right]= & \sum_{\text {pro }} \frac{d_{2}}{1+\delta_{34}} \frac{1}{2 \epsilon_{\mathbf{p}_{1}}} \prod_{i=2}^{4} \int d \Gamma_{i}(2 \pi)^{4} \delta^{4}\left(P_{\text {tot }} 4\right)\left|\mathcal{M}^{\text {pro }}\right|^{2} \\
& \times\left[\bar{f}_{1}^{0} \bar{f}_{2}^{0}\left(1 \pm \bar{f}_{3}^{0}\right)\left(1 \pm \bar{f}_{4}^{0}\right)\right] \beta \\
& \times\left(\chi_{3}\left(\mathbf{p}_{3}\right)+\chi_{4}\left(\mathbf{p}_{4}\right)-\chi_{2}\left(\mathbf{p}_{2}\right)-\chi_{1}\left(\mathbf{p}_{1}\right)\right) .
\end{aligned}
$$

Since the response function $\chi_{i}\left(\mathbf{p}_{i}\right)$ is an odd function of $\mathbf{p}_{i}$ for (anti-)quarks or is zero for gluons, whereas other integrand is an even function of $\mathbf{p}_{i}$, the result of the integral related to $\chi_{j}\left(\mathbf{p}_{i}\right)(i=2,3,4)$ is zero. Using relaxation time approximation $C\left[f_{1}\right]=-\delta f_{1} / \tau_{1}=-\beta \bar{f}_{1}^{0}\left(1 \pm \bar{f}_{1}^{0}\right) \chi_{1}\left(\mathbf{p}_{1}\right) / \tau_{1}$, we can obtain momentum dependent thermal relaxation time of species $a_{1}$, namely,

$$
\begin{aligned}
\tau_{1}^{-1}= & \sum_{\text {pro }} \frac{d_{2}}{\delta_{34}+1} \int \frac{d^{3} \mathbf{p}_{2}}{(2 \pi)^{3}} \frac{\bar{f}_{2}^{0}\left(1 \pm \bar{f}_{3}^{0}\right)\left(1 \pm \bar{f}_{4}^{0}\right)}{\left(1 \pm \bar{f}_{1}^{0}\right)} \\
& \times \int d t \frac{d \sigma^{\text {pro }}}{d t}
\end{aligned}
$$

where $\frac{d \sigma^{\text {pro }}}{d t}=\frac{\left\langle\left|M^{\text {pro }}\right|^{2}\right\rangle}{16 \pi s^{2}}$ denotes the differential scattering cross section with respect to the Mandelstam variables $s, t$, $u$.

Due to the large angle scattering is the most efficient mechanism for the transport process in a plasma with longrange interaction [117-119], a phenomenological weight factor $\sin \theta^{2} / 2=2 t u / s^{2}(\theta$ is the scattering angle in the center of mass system) is introduced in Eq. (A3). Alternatively, when the momentum transfer $\left|\mathbf{p}_{1}-\mathbf{p}_{3}\right|=\left|\mathbf{p}_{2}-\mathbf{p}_{4}\right|$ is small or $t=\left|P_{1}-P_{3}\right|=\left|P_{2}-P_{4}\right| \ll \sqrt{s}$, we assume that $\bar{f}_{1}=\bar{f}_{3}$ and $\bar{f}_{2}=\bar{f}_{4}$ for the elastic scatterings, the thermal relaxation time can finally be rewritten as

$\tau_{1}^{-1}=\sum_{\text {pro }} \frac{d_{2}}{\delta_{34}+1} \int \frac{d^{3} \mathbf{p}_{2}}{(2 \pi)^{3}} \bar{f}_{2}^{0}\left(1 \pm \bar{f}_{4}^{0}\right) \int d t \frac{d \sigma^{\text {pro }}}{d t} \frac{2 t u}{s^{2}}$.

The integration in terms of $t$-channel only has the logarithmic infrared divergence [119]. And this divergence can be regulated by restricting the $t$-channel integration from $-s$ to $-\mu_{D}^{2}$, where $\mu_{D}^{2}=g^{2} T^{2}$ is the infrared regulator in the upper bound of the $t$ integration [119].

\section{Appendix B}

Apart from $q+\bar{q} \rightarrow g$ process, another dominant process in the LLL approximation with the specific regime $m_{q}^{2} \ll$ $\alpha_{s, B} e B \ll T^{2} \ll e B$ is $t$ channel $q(P)+\bar{q}\left(P^{\prime \prime}\right) \rightarrow$ $q\left(P^{\prime}\right)+\bar{q}\left(P^{\prime \prime \prime}\right)$ scattering. The associated collision term has been presented by K.Hattori et al in Ref. [41] using the leading order perturbative QCD approach. We extend their result to nonzero quark chemical potential case, which has the following form

$$
\begin{aligned}
& C\left[f_{B, q}\left(p_{z}, \mu_{q}\right)\right]_{2 \rightarrow 2} \\
= & 2 g^{4} T_{R} C_{R}\left(\frac{\left|q_{f} e B\right|}{2 \pi}\right)\left(16 m_{f}^{4}\right) \\
\times & \frac{1}{\left(2 \epsilon_{f, 0}\right)^{2}} \beta \bar{f}_{B, q}^{0}\left(p_{z}, \mu_{q}\right)\left(1-\bar{f}_{B, \bar{q}}^{0}\left(p_{z}, \mu_{\bar{q}}\right)\right) \\
\times & \int \frac{d p_{z}^{\prime}}{2 \pi} \frac{1}{\left(\epsilon_{f, 0}^{\prime}\right)^{2}} \frac{\epsilon_{f, 0} \epsilon_{f, 0}^{\prime}}{\left|\epsilon_{f, 0} p_{z}^{\prime}-\epsilon_{f, 0}^{\prime} p_{z}\right|} \\
\times & \int \frac{d^{2} \mathbf{q}_{\perp}}{(2 \pi)^{2}} e^{-\frac{\mathbf{q}_{\perp}^{2}}{e B}} \frac{1}{\left(q^{2}+\operatorname{Re} \Pi_{z}(q)+i \operatorname{Im} \Pi_{z}(q)\right)^{2}} \\
& \times \bar{f}_{B, \bar{q}}^{0}\left(p_{z}^{\prime}, \mu_{\bar{q}}\right)\left(1-\bar{f}_{B, q}^{0}\left(p_{z}^{\prime}, \mu_{q}\right)\right)\left(\chi_{q}\left(p_{z}^{\prime}\right)-\chi_{q}\left(p_{z}\right)\right),
\end{aligned}
$$

where $\mathbf{q}=\mathbf{p}^{\prime}-\mathbf{p}=\mathbf{p}^{\prime \prime}-\mathbf{p}^{\prime \prime \prime}$ is the momentum transfer, $q^{2}=\mathbf{q}_{\perp}^{2}-q_{\|}^{2}$ and $-q_{\|}^{2}=-\left(P^{\prime}-P\right)_{\|}^{2}=2\left(\epsilon_{f, 0} \epsilon_{f, 0}^{\prime}-p_{z} p_{z}^{\prime}-\right.$ $\left.m_{f}^{2}\right) . \epsilon_{f, 0}=\sqrt{p_{z}^{2}+m_{f}^{2}}$ and $\epsilon_{f, 0}^{\prime}=\sqrt{p_{z}^{\prime 2}+m_{f}^{2}} \cdot \operatorname{Re} \Pi_{z}(q)$ and $\operatorname{Im} \Pi_{z}(q)$ in Eq. (B1) are the real and imaginary parts of gluon self-energy along the direction of magnetic field, respectively. In the static limit $\left(q_{0} \rightarrow 0\right), \operatorname{Re} \Pi_{\mathrm{z}}$ is the Debye mass $m_{D, B}$. In the work of Hasan et al. [50], the imaginary part of gluon self-energy in the strong magnetic field is given as

$$
\left.\frac{\operatorname{Im} \Pi_{z}(\mathbf{q})}{q_{0}}\right|_{q_{0} \rightarrow 0}=-\frac{g^{2}}{\mathbf{q}} \frac{\pi T^{2}}{2}-\frac{g^{2}}{q_{z}^{2}} \frac{\sum_{f} m_{f}^{2}\left|q_{f} e B\right|}{8 \pi T} .
$$

Thus we can note that $\operatorname{Im} \Pi_{z}(\mathbf{q})$ vanishes in the static limit. Furthermore, in chirality nonflip case $p_{z} \cdot p_{z}^{\prime}>0, \mid \epsilon_{f, 0} p_{z}^{\prime}-$ $\epsilon_{f, 0}^{\prime} p_{z} \mid$ and $-q_{\|}^{2}$ can rewritten as [41],

$$
\left|\epsilon_{f, 0} p_{z}^{\prime}-\epsilon_{f, 0}^{\prime} p_{z}\right|=\frac{m_{f}^{2}\left|p_{z}^{\prime 2}-p_{z}^{2}\right|}{\left|\epsilon_{f, 0} p_{z}^{\prime}+\epsilon_{f, 0}^{\prime} p_{z}\right|} \approx \frac{m_{f}^{2}}{\epsilon_{f, 0}}\left|p_{z}^{\prime}-p_{z}\right|
$$


and

$$
\begin{aligned}
-q_{\|}^{2}= & 2\left(\epsilon_{f, 0} \epsilon_{f, 0}^{\prime}-p_{z} p_{z}^{\prime}-m_{f}^{2}\right)=\frac{2 m_{f}^{2}\left(p_{z}^{\prime}-p_{z}\right)^{2}}{\epsilon_{f, 0} \epsilon_{f, 0}^{\prime}+p_{z} p_{z}^{\prime}+m_{f}^{2}} \\
& \approx \frac{m_{f}^{2}}{\epsilon_{f, 0}^{2}}\left(p_{z}^{\prime}-p_{z}\right)^{2}
\end{aligned}
$$

respectively. In the hierarchy of scale $e B \gg T^{2}$, the form factor $e^{-\frac{\mathbf{q}_{\perp}^{2}}{e B}}$ can reasonably be neglected due to $\mathbf{q}_{\perp}^{2} \sim-q_{\|}^{2} \lesssim$ $T^{2} \ll e B$, and $m_{D, B}^{2}$ always dominates over $q_{\|}^{2}$ in the regime $\alpha_{s, B} e B \gg m_{q}^{2}$. Therefore, Eq. (B1) can further reduce to

$$
\begin{aligned}
C & {\left[f_{B, q}\left(p_{z}, \mu_{q}\right)\right]_{2 \rightarrow 2} } \\
= & 8 \pi \alpha_{s}^{2} T_{R} C_{R}\left(\frac{e B}{2 \pi}\right) \frac{m_{f}^{2} \beta}{\epsilon_{f, 0}} \bar{f}_{B, q}^{0}\left(p_{z}, \mu_{q}\right) \\
(1 & \left.-\bar{f}_{B, \bar{q}}^{0}\left(p_{z}, \mu_{\bar{q}}\right)\right) \\
& \times \int \frac{d p_{z}^{\prime}}{2 \pi} \frac{1}{\left|p_{z}^{\prime}-p_{z}\right| \epsilon_{f, 0}^{\prime} / \epsilon_{f, 0}} \\
& \times \frac{1}{m_{D, B}^{2}} \bar{f}_{B, \bar{q}}^{0}\left(p_{z}^{\prime}, \mu_{\bar{q}}\right) \\
& \times\left(1-\bar{f}_{B, q}^{0}\left(p_{z}^{\prime}, \mu_{q}\right)\right)\left(\chi_{q}\left(p_{z}^{\prime}\right)-\chi_{q}\left(p_{z}\right)\right) .
\end{aligned}
$$

For the small $p_{z}-p_{z}^{\prime}, \chi_{q}\left(p_{z}^{\prime}\right)-\chi_{q}\left(p_{z}\right)$ can be approximated as

$$
\begin{aligned}
\left(\chi_{q}\left(p_{z}^{\prime}\right)-\chi_{q}\left(p_{z}\right)\right) & \approx\left(p_{z}^{\prime}-p_{z}\right) \partial_{p_{z}} \chi_{q}\left(p_{z}\right) \\
& =-\left(p_{z}^{\prime}-p_{z}\right) \partial_{p_{z}^{\prime}} \chi_{q}\left(p_{z}^{\prime}\right) .
\end{aligned}
$$

Therefore, the collision term for $2 \rightarrow 2$ process when $m_{q}^{2} \ll$ $\alpha_{s} e B$ is given by

$$
\begin{aligned}
C\left[f_{B, q}\left(p_{z}, \mu_{q}\right)\right]_{2 \rightarrow 2}= & -2 \alpha_{s, B}^{2} T_{R} C_{R}\left(\frac{e B}{\pi}\right) \frac{m_{f}^{2} \beta}{E_{f, 0} m_{D, B}^{2}} \\
& \times \bar{f}_{B, q}^{0}\left(p_{z}, \mu_{q}\right)\left(1-\bar{f}_{B, q}^{0}\left(p_{z}, \mu_{q}\right)\right) \\
& \times \bar{f}_{B, \bar{q}}^{0}\left(p_{z}, \mu_{\bar{q}}\right)\left(1-\bar{f}_{B, \bar{q}}^{0}\left(p_{z}, \mu_{\bar{q}}\right)\right) \\
& \times \chi_{q}\left(p_{z}\right) .
\end{aligned}
$$

In the LLL approximation with the hierarchy of scale $e B \gg$ $T^{2}, m_{D, B}^{2} \approx \sum_{f} \frac{\alpha_{s, B}\left|q_{f} e B\right|}{\pi}=2 \alpha_{s, B} T_{R} C_{R}\left(\frac{e B}{\pi}\right)$. Using $C\left[f_{B, q}\right]=-\beta \bar{f}_{B, q}^{0}\left(1+\bar{f}_{B, q}^{0}\right) \chi_{q} / \tau_{B, q}$, we finally get thermal relaxation time of (anti-)quarks for $f$ th flavor for $2 \rightarrow 2$ process,

$$
\begin{aligned}
\left.\frac{1}{\tau_{B, q(\bar{q})}}\right|_{2 \rightarrow 2}= & \alpha_{s, B} \frac{m_{q}^{2}}{\epsilon_{f, 0}} \bar{f}_{\bar{q}(q)}^{0}\left(p_{z}, \mu_{\bar{q}(q)}\right) \\
& \left(1-\bar{f}_{\bar{q}(q)}^{0}\left(p_{z}, \mu_{\bar{q}(q)}\right)\right) .
\end{aligned}
$$

In the anisotropic medium induced by strong magnetic field, the thermal relaxation time associated with the anisotropic parameter $\left(\xi^{\prime}\right)$ can be obtained by straightforwardly substituting $\bar{f}_{B, q}^{0}$ and $\alpha_{s, B}$ in Eq. (B8) with $\bar{f}_{B, q}^{\xi^{\prime}}$ and $\alpha_{e f f, B}$, respectively.

\section{References}

1. M. Cheng et al., Phys. Rev. D 74, 054507 (2006)

2. Y. Aoki, Z. Fodor, S.D. Katz, K.K. Szabo, Phys. Lett. B 643, 46 (2006)

3. J. Rafelski, B. Muller, Phys. Rev. Lett. 36, 517 (1976)

4. K. Tuchin, Adv. High Energy Phys. 2013, 490495 (2013)

5. V. Skokov, A.Y. Illarionov, V. Toneev, Int. J. Mod. Phys. A 24, 5925 (2009)

6. A. Bzdak, V. Skokov, Phys. Lett. B 710, 171 (2012)

7. W.T. Deng, X.G. Huang, Phys. Rev. C 85, 044907 (2012)

8. D.E. Kharzeev, L.D. McLerran, H.J. Warringa, Nucl. Phys. A 803, 227 (2008)

9. V. Voronyuk, V.D. Toneev, W. Cassing, E.L. Bratkovskaya, V.P. Konchakovski, S.A. Voloshin, Phys. Rev. C 83, 054911 (2011)

10. K. Tuchin, Phys. Rev. C 83, 017901 (2011)

11. L. McLerran, V. Skokov, Nucl. Phys. A 929, 184 (2014)

12. K. Fukushima, D.E. Kharzeev, H.J. Warringa, Phys. Rev. D 78, 074033 (2008)

13. J. Liao, Nucl. Phys. A 956, 99 (2016)

14. D.E. Kharzeev, H.U. Yee, Phys. Rev. D 83, 085007 (2011)

15. Y. Burnier, D.E. Kharzeev, J. Liao, H.U. Yee, Phys. Rev. Lett. 107, 052303 (2011)

16. E.J. Ferrer, V. de la Incera, X.J. Wen, Phys. Rev. D 91(5), 054006 (2015)

17. L. Yu, H. Liu, M. Huang, Phys. Rev. D 90(7), 074009 (2014)

18. H.L. Chen, K. Fukushima, X.G. Huang, K. Mameda, Phys. Rev. D 93(10), 104052 (2016)

19. D.M. Rodrigues, D. Li, E. FolcoCapossoli, H. Boschi-Filho, Phys. Rev. D 98(10), 106007 (2018)

20. D. Li, M. Huang, Y. Yang, P.H. Yuan, JHEP 1702, 030 (2017)

21. V.P. Pagura, D. Gomez Dumm, S. Noguera, N.N. Scoccola, Phys. Rev. D 95(3), 034013 (2017)

22. S. Mao, Phys. Lett. B 758, 195 (2016)

23. K. Fukushima, K. Hattori, H.U. Yee, Y. Yin, Phys. Rev. D 93(7), 074028 (2016)

24. S.K. Das, S. Plumari, S. Chatterjee, J. Alam, F. Scardina, V. Greco, Phys. Lett. B 768, 260 (2017)

25. K. Hattori, X.G. Huang, Nucl. Sci. Tech. 28(2), 26 (2017)

26. D. Dudal, T.G. Mertens, Phys. Rev. D 97(5), 054035 (2018)

27. M. Kurian, S.K. Das, V. Chandra, Phys. Rev. D 100(7), 074003 (2019)

28. Z.R. Zhu, S.Q. Feng, Y.F. Shi, Y. Zhong, Phys. Rev. D 99(12), 126001 (2019)

29. K.A. Mamo, Phys. Rev. D 94(4), 041901 (2016)

30. A.I. Akhiezer, V.B. Berestetsky, Quantum Electrodynamics (Interscience, New York, 1965)

31. V.P. Gusynin, V.A. Miransky, I.A. Shovkovy, Nucl. Phys. B 462, 249 (1996)

32. J.O. Andersen, W.R. Naylor, A. Tranberg, Rev. Mod. Phys. 88, 025001 (2016)

33. K. Fukushima, Y. Hidaka, Phys. Rev. Lett. 120(16), 162301 (2018)

34. B. Feng, Phys. Rev. D 96(3), 036009 (2017)

35. L. Thakur, P.K. Srivastava, Phys. Rev. D 100(7), 076016 (2019)

36. A. Das, H. Mishra, R.K. Mohapatra, Phys. Rev. D 101(3), 034027 (2020)

37. S. Li, H.U. Yee, Phys. Rev. D 97(5), 056024 (2018)

38. A. Das, H. Mishra, R.K. Mohapatra, Phys. Rev. D 99(9), 094031 (2019) 
39. A. Das, H. Mishra, R.K. Mohapatra, Phys. Rev. D 100(11), 114004 (2019)

40. K. Hattori, D. Satow, Phys. Rev. D 94(11), 114032 (2016)

41. K. Hattori, S. Li, D. Satow, H.U. Yee, Phys. Rev. D 95(7), 076008 (2017)

42. M. Kurian, V. Chandra, Phys. Rev. D 96(11), 114026 (2017)

43. A.N. Tawfik, A.M. Diab, T.M. Hussein, Int. J. Adv. Res. Phys. Sci. 3, 4 (2016)

44. M. Kurian, S. Mitra, S. Ghosh, V. Chandra, Eur. Phys. J. C 79(2), 134 (2019)

45. S.I. Nam, C.W. Kao, Phys. Rev. D 87(11), 114003 (2013)

46. K. Hattori, X.G. Huang, D.H. Rischke, D. Satow, Phys. Rev. D 96(9), 094009 (2017)

47. M. Kurian, V. Chandra, Phys. Rev. D 97(11), 116008 (2018)

48. B. Singh, L. Thakur, H. Mishra, Phys. Rev. D 97(9), 096011 (2018)

49. B. Singh, S. Mazumder, H. Mishra, JHEP 2005, 068 (2020)

50. M. Hasan, B.K. Patra, B. Chatterjee, P. Bagchi, Nucl. Phys. A 995, $121688(2020)$

51. S. Li, K.A. Mamo, H.U. Yee, Phys. Rev. D 94(8), 085016 (2016)

52. M. Kurian, V. Chandra, Phys. Rev. D 99, 116018 (2019)

53. C. Hoyos, F. Peña-Benitez, P. Witkowski, JHEP 1908, 146 (2019)

54. R.T. Delves, Rep. Prog. Phys. 28, 249 (1965)

55. Y. Hasegawa, T. Komine, Y. Ishikawa, A. Suzuki, H. Shirai, Jpn. J. Appl. Phys. 43, 35 (2004)

56. X.Z. Yan, C.S. Ting, Phys. Rev. B 81, 155457 (2010)

57. R. Ma, L. Zhu, L. Sheng, M. Liu, D.N. Sheng, Phys. Rev. B 84, 075420 (2011)

58. J.G. Checkelsky, N.P. Ong, Phys. Rev. B 80, 081413 (2008)

59. I. Mandal, K. Saha, Phys. Rev. B 101(4), 045101 (2020)

60. R. Lundgren, P. Laurell, G.A. Fiete, Phys. Rev. B 90(16), 165115 (2014)

61. J.R. Bhatt, A. Das, H. Mishra, Phys. Rev. D 99(1), 014015 (2019)

62. A. Das, H. Mishra, R.K. Mohapatra, Phys. Rev. D 102(1), 014030 (2020)

63. K.Y. Kim, K.K. Kim, Y. Seo, S.J. Sin, JHEP 1507, 027 (2015)

64. D. Dey, B.K. Patra, Phys. Rev. D 102(9), 096011 (2020)

65. P.K. Srivastava, B.K. Patra, Eur. Phys. J. A 53(6), 116 (2017)

66. L. Thakur, P.K. Srivastava, G.P. Kadam, M. George, H. Mishra, Phys. Rev. D 95(9), 096009 (2017)

67. P.K. Srivastava, L. Thakur, B.K. Patra, Phys. Rev. C 91(4), 044903 (2015)

68. A. Kumar, M.Y. Jamal, V. Chandra, J.R. Bhatt, Phys. Rev. D 97(3), 034007 (2018)

69. M.Y. Jamal, S. Mitra, V. Chandra, Phys. Rev. D 95(9), 094022 (2017)

70. P. Romatschke, M. Strickland, Phys. Rev. D 70, 116006 (2004)

71. P. Romatschke, M. Strickland, Phys. Rev. D 68, 036004 (2003)

72. A. Dumitru, Y. Guo, A. Mocsy, M. Strickland, Phys. Rev. D 79, 054019 (2009)

73. M. Margotta, K. McCarty, C. McGahan, M. Strickland, D. YagerElorriaga, Phys. Rev. D 83, 105019 (2011).

74. M.Y. Jamal, I. Nilima, V. Chandra, V.K. Agotiya, Phys. Rev. D 97(9), 094033 (2018)

75. S. Biondini, N. Brambilla, M.A. Escobedo, A. Vairo, Phys. Rev. D 95(7), 074016 (2017)

76. L. Thakur, N. Haque, U. Kakade, B.K. Patra, Phys. Rev. D 88(5), 054022 (2013)

77. S. Rath, B.K. Patra, Phys. Rev. D 100(1), 016009 (2019)

78. S. Rath, B.K. Patra, Phys. Rev. D 102(3), 036011 (2020)

79. S. Rath, B.K. Patra, JHEP 1712, 098 (2017)

80. A. Ayala, C.A. Dominguez, S. Hernandez-Ortiz, L.A. Hernandez, M. Loewe, D.M. Paret, R. Zamora, Phys. Rev. D 98(3), 031501 (2018)

81. A.V. Smilga, Phys. Rev. D 45, 1378 (1992)
82. N. Ashcroft, N. Mermin, Solid State Physics, HRW International Editions (Holt, Rinehart and Winston, New York, 1976)

83. K. Seeger, Semiconductor Physics, 5th edn. (Springer, Berlin, 1990)

84. C. Caroli, K. Maki, Phys. Rev. 164, 591 (1967)

85. J.M. Ziman, Principles of the Theory of Solids (Cambridge University Press, Cambridge, 1964)

86. E.N. Adams, T.D. Holstein, J. Phys. Chem. Solids 10, 254 (1959)

87. F.M. Hashimzade, K.A. Hasanov, B.H. Mehdiyev, S. Cakmak, Phys. Scr. 81, 015701 (2010)

88. C.R. Wang, W.S. Lu, W.L. Lee, Phys. Rev. B 82, 121406(R) (2010)

89. A. Kundu, M.A. Alrefae, T.S. Fisher, J. Appl. Phys. 121, 125113 (2017)

90. A.F. May, G.J. Snyder, Introduction to modeling thermoelectric transport at high temperatures, chapter 11, in Thermoelectrics and Its Energy Harvesting, vol. 1, ed. by D.M. Rowe (CRC Press, Boca Raton, 2012)

91. H.J. Goldsmid, Thermoelectric Refrigeration (Plenum Press, New York, 1964)

92. M. Matusiak, M. Babij, T. Wolf, Phys. Rev. B 97, 100506 (2018)

93. T.M. Tritt, M.A. Subramanian, G. Editors, MRS Bull. 31(3), 188198 (2006)

94. A. Harutyunyan, A. Sedrakian, Phys. Rev. C 94(2), 025805 (2016)

95. S. Lin, L. Yang, Phys. Rev. D 101(3), 034006 (2020)

96. S. Chakrabarty, Phys. Rev. D 54, 1306 (1996)

97. M.L. Bellac, Thermal Field Theory (Cambridge University Press, Cambridge, 1996)

98. P.F. Kelly, Q. Liu, C. Lucchesi, C. Manuel, Phys. Rev. Lett. 72, 3461 (1994)

99. P.F. Kelly, Q. Liu, C. Lucchesi, C. Manuel, Phys. Rev. D 50, 4209 (1994)

100. J.P. Blaizot, E. Iancu, Phys. Rep. 359, 355 (2002)

101. M. Hasan, B. Chatterjee, B.K. Patra, Eur. Phys. J. C 77(11), 767 (2017)

102. E. Braaten, R.D. Pisarski, Phys. Rev. D 42, 2156 (1990)

103. E. Braaten, R.D. Pisarski, Phys. Rev. Lett. 64, 1338 (1990)

104. R.D. Pisarski, Nucl. Phys. A 525, 175 (1991)

105. R.D. Pisarski, Phys. Rev. D 47, 5589 (1993)

106. E.V. Shuryak, Sov. Phys. JETP 47, 212 (1978)

107. E.V. Shuryak, Zh. Eksp, Teor. Fiz. 74, 408 (1978)

108. A. Bazavov, N. Brambilla, X. Garcia i Tormo, P. Petreczky, J. Soto, A. Vairo, Phys. Rev. D 86, 114031 (2012)

109. J.F. Owens, Rev. Mod. Phys. 59, 465 (1987)

110. R. Cutler, D.W. Sivers, Phys. Rev. D 17, 196 (1978)

111. A. Bandyopadhyay, B. Karmakar, N. Haque, M.G. Mustafa, Phys. Rev. D 100(3), 034031 (2019)

112. H.-T. Ding, A. Francis, O. Kaczmarek, F. Karsch, E. Laermann, W. Soeldner, Phys. Rev. D 83, 034504 (2011)

113. A. Amato, G. Aarts, C. Allton, P. Giudice, S. Hands, J.I. Skullerud, Phys. Rev. Lett. 111(17), 172001 (2013)

114. B.B. Brandt, A. Francis, B. Jäger, H.B. Meyer, Phys. Rev. D 93(5), 054510 (2016)

115. H.A. Weldon, Phys. Rev. D 26, 1394 (1982)

116. S.R. De Groot, W.A. van Leeuwen, Ch.G. van Weert, Relativistic Kinetic Theory (North-Holland, Amsterdam, 1980)

117. E.M. Lifshitz, L.P. Pitaevskii, Physical Kinetics (Pergamon Press, New York, 1981)

118. P. Zhuang, J. Hufner, S.P. Klevansky, L. Neise, Phys. Rev. D 51, 3728 (1995)

119. M.H. Thoma, Phys. Rev. D 49, 451 (1994) 